Pacific

Journal of

Mathematics

\title{
APPLICATIONS OF THE DEFORMATION FORMULA OF HOLOMORPHIC ONE-FORMS
}

Quanting Zhao And Sheng RaO 


\title{
APPLICATIONS OF THE DEFORMATION FORMULA OF HOLOMORPHIC ONE-FORMS
}

\author{
QuANTING ZhaO AND SHENG RAO
}

\begin{abstract}
This paper studies some geometric aspects of moduli of curves $M_{g}$, using as a tool the deformation formula of holomorphic one-forms. Quasi-isometry guarantees the $L^{2}$ convergence of deformation of holomorphic one-forms, which is a kind of global result. After giving the period map a full expansion, we can also write out the Siegel metric, curvature and second fundamental form of a nonhyperelliptic locus of $M_{g}$ in a quite detailed manner, while gaining some understanding of a totally geodesic manifold in a nonhyperelliptic locus.
\end{abstract}

\section{Introduction}

This paper is a complement to our joint paper [Liu et al. 2012b] with Kefeng Liu, and explores more applications of the deformation formula of holomorphic oneforms to some problems related to moduli spaces of Riemann surfaces, including the full expansion of the period map, the Siegel metric and its curvature formulae, the second fundamental form of Torelli space's nonhyperelliptic locus, and also a global result about the deformation of holomorphic one-forms.

We start with the Kuranishi coordinate of the Teichmüller space $\mathscr{T}_{g}$ of Riemann surfaces of genus $g$ and the deformation formula of holomorphic one-forms $\theta(t)$, whose construction is contained in Section 2. The key points of the deformation formula lie in Theorem 2.1. To be more precise, on the Kuranishi family $\varpi: \mathscr{L} \rightarrow \Delta$ with a Riemann surface $\varpi^{-1}(0)=X_{0}$ as its central fiber and a global holomorphic one-form of the central fiber $\theta \in H^{0}\left(X_{0}, \Omega_{X_{0}}^{1}\right)$, the deformation formula of holomorphic one-forms emerges as

$$
\left.\theta(t)=\theta+\sum_{|I| \geq 1} t^{I}\left(\sum_{j=1}^{n} \mathbb{M}\left(\mu_{j}\right\lrcorner \eta_{\left(i_{1}, \ldots, i_{j}-1, \ldots, i_{n}\right)}\right)\right),
$$

Rao is the corresponding author and is partially supported by the 52nd China Postdoctoral Science Foundation, No. 2012M521157, and the National Natural Science Foundation of China, No. 11301477. MSC2010: primary 32G15; secondary 32G20, 30F30.

Keywords: moduli of Riemann surfaces, Teichmüller theory, period matrices, variation of Hodge structures, differentials on Riemann surfaces. 
such that $\theta(t) \in H^{0}\left(X_{t}, \Omega_{X_{t}}^{1}\right)$, where

$$
\mu(t)=\sum_{i=1}^{n} t_{i} \mu_{i}
$$

is the integrable Beltrami differential of the Kuranishi family and $\mathbb{W}$ is the harmonic projector in Hodge decomposition with respect to the Poincaré metric on $X_{0}$. Moreover, $\mathbb{G}$ denotes the associated Green operator, and $\eta_{I}$ is given by

$$
\left\{\begin{array}{l}
\left.\eta_{i}=-\mathbb{G} \bar{\partial}^{*} \partial\left(\mu_{i}\right\lrcorner \theta\right), \\
\left.\eta_{\left(i_{1}, \ldots, i_{n}\right)}=-\mathbb{G} \bar{\partial}^{*} \partial\left(\sum_{k=1}^{n} \mu_{k}\right\lrcorner \eta_{\left(i_{1}, \ldots, i_{k}-1, \ldots, i_{n}\right)}\right) .
\end{array}\right.
$$

We identify $\eta_{i}$ with $\eta_{\left(0, \ldots, 1_{i-\text { th }}, \ldots, 0\right)}$ here. Apply (1-1) to the canonical basis $\left\{\theta_{p}^{\alpha}\right\}_{\alpha=1}^{g}$ of $H^{0}\left(X_{p}, \Omega_{X_{p}}^{1}\right)$ with respect to the symplectic basis

$$
\left\{A_{\gamma}, B_{\gamma}\right\}_{\gamma=1}^{g}
$$

for the Kuranishi coordinate $\Delta_{p, \epsilon}$, yielding

$$
\begin{aligned}
\left.\theta_{p}^{\alpha}(t)=\theta_{p}^{\alpha}+\sum_{i=1}^{n} t_{i}\left(\mathbb{H}\left(\mu_{i}\right\lrcorner \theta_{p}^{\alpha}\right)+d f_{i}^{\alpha}\right) \\
\left.+\sum_{|I| \geq 2} t^{I}\left(\sum_{j=1}^{n} \mathbb{M}\left(\mu_{j}\right\lrcorner \eta_{\left(i_{1}, \ldots, i_{j}-1, \ldots, i_{n}\right)}^{\alpha}\right)+d f_{j,\left(i_{1}, \ldots, i_{j}-1, \ldots, i_{n}\right)}^{\alpha}\right) .
\end{aligned}
$$

We then define $A(t)$ by

$$
\left.\sum_{|I| \geq 1} t^{I}\left(\sum_{j=1}^{n} \mathbb{H}\left(\mu_{j}\right\lrcorner \eta_{\left(i_{1}, \ldots, i_{j}-1, \ldots, i_{n}\right)}^{\alpha}\right)\right)=A(t)_{\beta}^{\alpha} \bar{\theta}_{p}^{\beta} .
$$

Let $\sigma_{p}, \pi_{p}$ be the $A, B$ period matrices of $\left\{\theta_{p}^{\alpha}\right\}_{\alpha=1}^{g}$ and $M_{p}=\operatorname{Im}\left(\pi_{p}\right)$. The period map

$$
\Pi: \mathscr{T}_{g} \rightarrow \mathscr{H}_{g}
$$

where $\mathscr{H}_{g}$ is the classifying space of Hodge structures of weight one, can be written out on the Kuranishi coordinate as

$$
\Pi(t)=\left(\pi_{p}+\bar{\pi}_{p} A(t)^{T}\right)\left(\mathbb{1}_{g}+A(t)^{T}\right)^{-1} .
$$

H. Rauch [1959] and A. Mayer [1969] have expanded the period map only up to the first order, while Fangliang Yin's expansion formula [2010] via computing high derivatives of the period map is not explicit for orders larger than two, since it is difficult to write all the derivatives out. In a different manner, by solving a recursive relation, we can get an explicit formula for every order part of the expansion: 
Theorem 1.1 (Theorem 2.5). The period map

$$
\Pi: \mathscr{T}_{g} \rightarrow \mathscr{H}_{g}
$$

has the following full expansion on the Kuranishi coordinate $\Delta_{p, \epsilon}$ :

$$
\begin{aligned}
& \left.\left.\Pi_{\alpha \beta}(t)=\Pi_{\alpha \beta}(0)+\int_{X_{0}} \theta^{\alpha} \wedge \mathbb{M}(\mu(t)\lrcorner \theta^{\beta}\right)+\int_{X_{0}} \theta^{\alpha} \wedge \mathbb{H}(\mu(t)\lrcorner \eta_{t, 1}^{\beta}\right) \\
& \left.\left.-\frac{\mathrm{i}}{2} \int_{X_{0}} \theta^{\alpha} \wedge \mathbb{H}(\mu(t)\lrcorner \theta^{\delta}\right) M^{\delta \gamma} \int_{X_{0}} \theta^{\gamma} \wedge \mathbb{H}(\mu(t)\lrcorner \theta^{\beta}\right) \\
& +\sum_{k \geq 3} \sum_{\substack{m_{i}>0,1 \leq i \leq l \\
m_{1}+\cdots+m_{l}=k}}\left\{(-1)^{l-1} \int_{X_{0}} \theta^{\alpha} \wedge \mathbb{M}(\mu(t)\lrcorner \eta_{t, m_{1}-1}^{\alpha_{1}}\right) \\
& \left.\times \frac{\mathrm{i}}{2} M^{\alpha_{1} \alpha_{2}} \int_{X_{0}} \theta^{\alpha_{2}} \wedge \mathfrak{H}(\mu(t)\lrcorner \eta_{t, m_{2}-1}^{\alpha_{3}}\right) \cdots \\
& \left.\left.\times \frac{\mathrm{i}}{2} M^{\alpha_{2 l-3} \alpha_{2 l-2}} \int_{X_{0}} \theta^{\alpha_{2 l-2}} \wedge \mathfrak{H}(\mu(t)\lrcorner \eta_{t, m_{l}-1}^{\beta}\right)\right\},
\end{aligned}
$$

where $\eta_{t, N}^{\alpha}$ is the $N$-th order part of the expansion of $\eta_{t}^{\alpha}$ in the deformation formula of $\theta^{\alpha}$ by Theorem 2.1, $M^{\alpha \beta}$ is the inverse matrix of $M_{p}=\operatorname{Im}\left(\pi_{p}\right)$, and $\mathrm{i}=\sqrt{-1}$.

Geometric information on the period map is contained in the homogeneous parts in this theorem, whose meaning will become apparent in the following sections.

In Section 3, by a quasi-isometry result in [Liu et al. 2012a] for the operator $\bar{\partial} \circ \mathbb{G} \circ \partial$, we obtain a global result for the deformation of holomorphic one-forms.

Proposition 1.2. The $(1,0)$-form $\eta(t)$ on $X_{p}$ constructed in Theorem 2.1 converges in $L^{2}$-norm as long as $|t|<1$, and so does $\theta(t)$ constructed in Theorem 2.1.

In Section 4, the deformation formula of holomorphic one-forms provides us with an effective way to write out the Siegel metric and its curvature explicitly according to the expansion degree of $t$. To maintain that the formula is integral and clean, we need Definition 4.1 of symmetric derivatives. Also the normal coordinate (4-5) is used in our calculation.

Theorem 1.3. The Siegel metric $\omega_{s}(t)$ on the nonhyperelliptic locus of the Torelli space Tor $_{g}$ can be written as

$$
\begin{aligned}
\omega_{S}(t) & =\frac{\mathrm{i}}{2} \sum_{n=1}^{\infty} \frac{1}{n} \partial \bar{\partial} \operatorname{tr}(A(t) \overline{A(t)})^{n} \\
& =\frac{\mathrm{i}}{2} \sum_{k \geq 0} \sum_{\substack{m_{i}>0,1 \leq i \leq 2 l \\
m_{1}+\cdots+m_{2 l}=k+2}} \frac{1}{l} \operatorname{tr}\left(S_{i \bar{j}}\left(A_{m_{1}}(t) \overline{A_{m_{2}}(t)} \ldots \overline{A_{m_{2 l}}(t)}\right)\right) d t_{i} \wedge d \bar{t}_{j},
\end{aligned}
$$


and its curvature $\mathrm{R}_{i \bar{j} k \bar{l}}$ is given by

$$
\begin{aligned}
& \mathrm{R}_{i \bar{j} k \bar{l}}=-\sum_{N \geq 0} \sum_{\substack{m_{i}>0,1 \leq i \leq 2 l \\
m_{1}+\cdots+m_{2 l}=N+4}} \frac{1}{l} \operatorname{tr}\left(S_{i \bar{j}} \boldsymbol{S}_{k \bar{l}}\left(A_{m_{1}}(t) \overline{A_{m_{2}}(t)} \ldots \overline{A_{m_{2 l}}(t)}\right)\right) \\
& +\sum_{N \geq 0} \sum_{\substack{N_{i} \geq 0,1 \leq i \leq 3 \\
\sum_{i=1}^{3} N_{i}=N}}\left[\sum_{\substack{m_{i}>0,1 \leq i \leq l \\
m_{1}+\cdots+m_{l}=N_{1}}}(-1)^{l} \prod_{i=1}^{l} \frac{1}{s_{i}}\right. \\
& \times \sum_{\substack{m_{1 n}>0,1 \leq n \leq 2 s_{1} \\
\sum_{n=1}^{2 s} 1}} \operatorname{tr}\left(S_{q \overline{i_{1}}}\left(A_{m_{11}}(t) \ldots \overline{A_{m_{12 s_{1}}}(t)}\right)\right) \\
& \times \sum_{\substack{m_{2 n}>0,1 \leq n \leq 2 s_{2} \\
\sum_{n=1}^{2 s_{2}} m_{2 n}=m_{2}+2}} \operatorname{tr}\left(S_{i_{1} \overline{i_{2}}}\left(A_{m_{21}}(t) \ldots \overline{A_{m_{22 s_{2}}}(t)}\right)\right) \cdots \\
& \left.\times \sum_{\substack{m_{l n}>0,1 \leq n \leq 2 s_{l} \\
\sum_{n=1}^{2 s_{l}} m_{l n}=m_{l}+2}} \operatorname{tr}\left(\boldsymbol{S}_{i_{l-1} \bar{p}}\left(A_{m_{l 1}}(t) \ldots \overline{A_{m_{l 2 s_{l}}}(t)}\right)\right)\right] \\
& \times\left[\sum_{\substack{m_{i}>0,1 \leq i \leq 2 l \\
\sum_{i=1}^{2 l} m_{i}=N_{2}+3}} \frac{1}{l} \operatorname{tr}\left(S_{i} S_{k \bar{q}}\left(A_{m_{1}}(t) \ldots \overline{A_{m_{2 l}}(t)}\right)\right)\right] \\
& \times\left[\sum_{\substack{m_{i}>0,1 \leq i \leq 2 l \\
\sum_{i=1}^{2 l}, m_{i}=N_{3}+3}} \frac{1}{l} \operatorname{tr}\left(S_{\bar{j}} S_{p \bar{l}}\left(A_{m_{1}}(t) \ldots \overline{A_{m_{2 l}}(t)}\right)\right)\right],
\end{aligned}
$$

where we need the convention that the first square bracket in the second summand equals $\delta_{q p}$ if $N_{1}=0$.

The bound $\mathrm{H}(v) \leq-2 / g$ for the holomorphic sectional curvature $\mathrm{H}(v)$ along the direction $v=\sum_{i=1}^{3 g-\overline{3}} a_{i} \mu_{i} \in \mathbb{H}_{\bar{\partial}}^{0,1}\left(X_{p}, T_{X_{p}}\right)$ of $\mathrm{R}_{i \bar{j} k \bar{l}}$ is also discussed in this section.

Section 5 is motivated by Oort's conjecture and Moonen's result as follows.

Conjecture 1.4 [Oort 1997]. Let $\overline{\mathscr{F}_{g}}:=\overline{\mathscr{F}\left(M_{g}\right)} \subset \mathscr{A}_{g}$ be the Zariski closure of the (open) Torelli locus $\mathscr{F}_{g}:=\mathscr{F}\left(\mathcal{M}_{g}\right)$. For $g \geq 4$, determine all special subvarieties (or varieties of Hodge type) of positive dimension in $\mathscr{A}_{g}$ that are contained in $\overline{\mathscr{F}_{g}}$ and meet $\mathscr{F}_{g}$. Conjecturally, there are no such subvarieties when $g$ is sufficiently large.

As a complex orbifold, $\mathscr{A}_{g}(\mathbb{C})$ is a quotient of the Siegel space, which is an irreducible homogeneous symmetric space under the group $\operatorname{Sp}(g, \mathbb{R})$, and special subvarieties can be considered as images of orbits of an algebraic subgroup. Here we refer the readers to the remarkable survey [Moonen and Oort 2013, Section 3.6] for the three equivalent definitions of special subvarieties and other preliminaries. 
Fortunately, we have an important result by B. Moonen [1995]: Let $V \subset \mathscr{A}_{g}$ be an algebraic subvariety. Then $V$ is a special subvariety if and only if it is totally geodesic with respect to the Siegel metric and it contains at least one special point. From [Oort 2003], we know that a special point in $\mathscr{A}_{g}$ corresponds to a moduli point of principally polarized abelian variety $(A, \lambda)$ with $A$ admitting sufficiently many complex multiplications. The notion of sufficiently many complex multiplications of an abelian variety $A$ has an equivalent expression: there is a commutative semisimple subalgebra $E \subset \operatorname{End}^{0}(A):=\operatorname{End}(A) \otimes_{\mathbb{Z}} \mathbb{Q}$ with $\operatorname{dim}_{\mathbb{Q}} E=2 g$. Perhaps we need a more geometric description of special points for deeper investigation.

Therefore, it is important to understand the second fundamental form of the Torelli locus and totally geodesic subvarieties, which is also proposed by B. Farb [2006], R. Hain [1999] and D. Toledo [1987]. By our deformation method, we can get the second fundamental form of a nonhyperelliptic locus and some understanding of a totally geodesic manifold in a nonhyperelliptic locus. The full formula for the second fundamental form is included in the Appendix, since it is long and complicated.

Proposition 1.5. The second fundamental form of a nonhyperelliptic locus at the central point is

$$
\Sigma_{i \bar{j} k \bar{l}}(0)=\operatorname{tr}\left(A_{i k} \overline{A_{j l}}\right),
$$

where $A_{i j}$ is defined in our discussion of the homogeneous part of $A(t)$ after Definition 2.2.

As a direct corollary, the holomorphic sectional curvature along a totally geodesic submanifold in a nonhyperelliptic locus of $\mathscr{T}_{g}$ is bounded from below. Moreover, we obtain the relationship between the total geodesicness and local symmetry, that is, a totally geodesic manifold in the nonhyperelliptic locus of $\mathscr{T}_{g}$ must be locally symmetric.

\section{Full expansion of the period map}

Kuranishi coordinates and small deformation of holomorphic one-forms. Fix a compact topological surface $\Sigma$ of genus $g$ with $g \geq 2$. The pair $(C,[f])$ is a Riemann surface $C$ with the Teichmüller structure $[f]$, where $f$ is an orientation-preserving homeomorphism from $C$ to $\Sigma$ and $[f]$ denotes the isotopic class represented by $f$. An isomorphism between two Riemann surfaces with the Teichmüller structures $(C,[f])$ and $\left(C^{\prime},\left[f^{\prime}\right]\right)$ is a biholomorphic map $\phi$ from $C$ to $C^{\prime}$ such that $[f]=\left[f^{\prime} \phi\right]$. The equivalence classes of all compact Riemann surfaces of genus $g$ with this Teichmüller structure, modulo the isomorphism equivalences, actually constitute $\mathscr{T}_{g}$. Thus an isomorphism class of $[C,[f]]$ is a point in $\mathscr{T}_{g}$.

From the construction of the Hilbert scheme, the existence of the Kuranishi family 
of Riemann surfaces follows. To be more precise, for every Riemann surface $C$, there exists a holomorphic deformation $(\varpi, \varphi)$

$$
\varpi: \mathscr{X} \rightarrow B, \quad \varphi: C \stackrel{\simeq}{\rightarrow} X_{b_{0}}
$$

of $C$ parametrized by a pointed base $\left(B, b_{0}\right)$ and a complex manifold with $\operatorname{dim}_{\mathbb{C}} B=$ $3 g-3$; this deformation is universal at $b_{0}$ and actually universal at every point $b$ of $B$. The pair $(\varpi, \varphi)$ is called the Kuranishi family of $C$. For any other deformation $(\iota, \psi)$

$$
\iota: \mathscr{X}^{\prime} \rightarrow B^{\prime}, \quad \psi: C \stackrel{\simeq}{\rightarrow} X_{b_{0}^{\prime}}^{\prime}
$$

of $C$, there exists a unique map $(\phi, \Phi)$ in a small neighborhood of $b_{0}^{\prime}$ such that the diagram

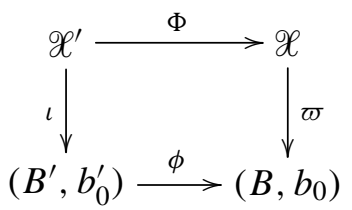

commutes, where $\varphi^{-1} \Phi_{b_{0}^{\prime}} \psi=\mathbb{1}_{C}$ and $\mathscr{X}^{\prime}$ is isomorphic to the pullback family $\Phi^{*} \mathscr{X}$ on the small neighborhood of $b_{0}^{\prime}$. Accordingly, we also have a family of Riemann surfaces with the Teichmüller structure $\left(X_{b},\left[f_{b}\right]\right)$, that is, $\varpi: \mathscr{X} \rightarrow B$, together with the local topological trivialization

$$
F^{\alpha}:\left.\mathscr{X}\right|_{U_{\alpha}} \rightarrow \Sigma \times U_{\alpha},
$$

where $\bigcup_{\alpha} U_{\alpha}$ is an open covering of $B$ such that $\left[F_{b}^{\alpha}\right]=\left[f_{b}\right]$ with $b \in U_{\alpha}$. For any Riemann surface with the Teichmüller structure $(C,[f])$, the Kuranishi family also exists and satisfies exactly analogous universal properties to the one without this Teichmüller structure. Possibly after shrinking $B$, we can describe the Kuranishi family of $(C,[f])$ as a triple $(\varpi, \varphi, F)$ given by

$$
\varpi: \mathscr{X} \rightarrow B, \quad \varphi: C \stackrel{\simeq}{\rightarrow} C_{b_{0}}, \quad F: \mathscr{X} \rightarrow \Sigma \times B,
$$

where $F$ is a topological trivialization such that $F_{b_{0}} \varphi=f$.

A Kuranishi coordinate chart of $\mathscr{T}_{g}$ is given by

$$
\left(B, b_{0}\right) \rightarrow \mathscr{T}_{g}, \quad t \rightarrow\left[X_{t},\left[F_{t}\right]\right],
$$

where the triple $(\varpi, \varphi, F)$ is the Kuranishi family of $(C,[f])$. By Ehresmann's classical theorem, there is a natural diffeomorphism $\Psi: X_{b_{0}} \times B \rightarrow \mathscr{X}$; all the fibers of $\varpi: \mathscr{X} \rightarrow B$

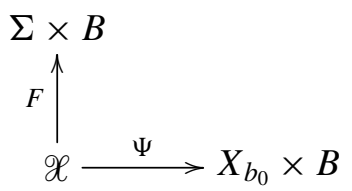


share the same differential structure as $X_{b_{0}}$. From this point of view, for every $b \in B$, the map $F_{b} \Psi_{b}^{-1}$ can be deformed to $F_{b_{0}} \Psi_{b_{0}}^{-1}$, that is,

$$
\left[F_{b} \Psi_{b}^{-1}\right]=\left[F_{b_{0}} \Psi_{b_{0}}^{-1}\right] .
$$

Let $\omega: H_{1}(\Sigma, \mathbb{Z}) \times H_{1}(\Sigma, \mathbb{Z}) \rightarrow \mathbb{Z}$ be the intersection pairing on $\Sigma$. The symplectic basis of $H_{1}(\Sigma, \mathbb{Z})$ on $(\Sigma, \omega)$ gives, from the map $\Psi F^{-1}$, one such basis on $X_{b_{0}}$, which is enjoyed by the whole Kuranishi family $\mathscr{L}$ over the Kuranishi coordinate chart $B$. Later on we will write $\left(B, b_{0}\right)$ as $\Delta_{p, \epsilon}$, where $p$ denotes the point $[C$, $[f]]$ in $\mathscr{T}_{g}$, and $\Delta_{p, \epsilon}=\left\{t \in \mathbb{C}^{n} \mid\|t\|<\epsilon, t(p)=0\right\}$ with $n=3 g-3$.

Let $\Delta_{p, \epsilon}$ be the Kuranishi coordinate centered at $p \in \mathscr{T}_{g}$ above. Denote the corresponding Kuranishi family on $\Delta_{p, \epsilon}$ by $\varpi: \mathscr{L} \rightarrow \Delta_{p, \epsilon}$ with the central fiber $\varpi^{-1}(0)=X_{p}$. Let

$$
\theta \in H^{0}\left(X_{p}, \Omega_{X_{p}}^{1}\right)
$$

be a global holomorphic one-form on $X_{p}$. We will construct $\theta(t) \in H^{0}\left(X_{t}, \Omega_{X_{t}}^{1}\right)$, a holomorphic deformation of $\theta$ with $t$ small.

Denote the well known Poincaré metric on $X_{p}$ by $\omega_{p}$. Fix $\left\{\mu_{i}\right\}_{i=1}^{n}$ as a basis of harmonic $T_{X_{p}}^{(1,0)}$-valued $(0,1)$ forms, written as $\mathbb{\boxplus}_{\bar{\partial}}^{0,1}\left(X_{p}, T_{X_{p}}^{(1,0)}\right)$, on $\left(X_{p}, \omega_{p}\right)$. And $\mu(t)=\sum_{i=1}^{n} t_{i} \mu_{i}$ is the integrable Beltrami differential of the Kuranishi family $\varpi: \mathscr{X} \rightarrow \Delta_{p, \epsilon}$.

Theorem 2.1 [Liu et al. 2012b, Theorem 2.1 and Corollary 2.2]. Given $\theta \in$ $H^{0}\left(X_{p}, \Omega_{X_{p}}^{1}\right)$, there exists a unique $(1,0)$-form $\eta(t)$ on $X_{p}$ that is holomorphic in $t$ for sufficiently small $t$, satisfying

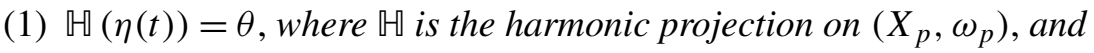

(2) $\theta(t)=(\mathbb{1}+\mu(t))\lrcorner \eta(t) \in H^{0}\left(X_{t}, \Omega_{X_{t}}^{1}\right)$,

and $\theta(t)$ is the desired deformation of $\theta$, given by

$$
\begin{aligned}
\left.\theta(t)=\theta+\sum_{i=1}^{n} t_{i}\left(\mathbb{H}\left(\mu_{i}\right\lrcorner \theta\right)+d f_{i}\right) \\
\left.\quad+\sum_{|I| \geq 2} t^{I}\left(\sum_{j=1}^{n} \mathbb{H}\left(\mu_{j}\right\lrcorner \eta_{\left(i_{1}, \ldots, i_{j}-1, \ldots, i_{n}\right)}\right)+d f_{j,\left(i_{1}, \ldots, i_{j}-1, \ldots, i_{n}\right)}\right),
\end{aligned}
$$

where $f_{j,\left(i_{1}, \ldots, i_{j}-1, \ldots, i_{n}\right)} \in C^{\infty}\left(X_{p}\right)$.

Based on this, an explicit formula of the period map and variation of Hodge structures on Kuranishi coordinates are discussed below. Denote the canonical basis of $H^{0}\left(X_{p}, \Omega_{X_{p}}^{1}\right)$ by $\left\{\theta_{p}^{\alpha}\right\}_{\alpha=1}^{g}$ with respect to the symplectic basis $\left\{A_{\gamma}, B_{\gamma}\right\}_{\gamma=1}^{g}$ for the Kuranishi coordinate $\Delta_{p, \epsilon}$. Let $\sigma_{p}, \pi_{p}$ be the $A, B$ period matrices of 
$\left\{\theta_{p}^{\alpha}\right\}_{\alpha=1}^{g}$ and $M_{p}=\operatorname{Im}\left(\pi_{p}\right)$. Applying the deformation formula above, we get the holomorphic one-forms $\theta_{p}^{\alpha}(t)$ on $X_{t}$, starting at $\theta_{p}^{\alpha}$, given by

$$
\begin{aligned}
\theta_{p}^{\alpha}(t)=\theta_{p}^{\alpha}+ & \left.\sum_{i=1}^{n} t_{i}\left(\mathbb{M}\left(\mu_{i}\right\lrcorner \theta_{p}^{\alpha}\right)+d f_{i}^{\alpha}\right) \\
& \left.+\sum_{|I| \geq 2} t^{I}\left(\sum_{j=1}^{n} \mathbb{H}\left(\mu_{j}\right\lrcorner \eta_{\left(i_{1}, \ldots, i_{j}-1, \ldots, i_{n}\right)}^{\alpha}\right)+d f_{j,\left(i_{1}, \ldots, i_{j}-1, \ldots, i_{n}\right)}^{\alpha}\right) .
\end{aligned}
$$

Definition 2.2 $(A(t)$ and $E(t))$. $A(t)$ is a $g \times g$ matrix, while $E(t)$ is a $g \times 1$ vector defined by

$$
\begin{gathered}
\left.\sum_{|I| \geq 1} t^{I} \sum_{j=1}^{n} \mathbb{H}(\mu j\lrcorner \eta_{\left(i_{1}, \ldots, i_{j}-1, \ldots, i_{n}\right)}^{\alpha}\right)=A(t)_{\beta}^{\alpha} \bar{\theta}_{p}^{\beta}, \\
\sum_{|I| \geq 1} t^{I}\left(\sum_{j=1}^{n} d f_{j,\left(i_{1}, \ldots, i_{j}-1, \ldots, i_{n}\right)}^{\alpha}\right)=E^{\alpha}(t) .
\end{gathered}
$$

We write the homogeneous part of order $N$ of $A(t)$ as $A_{N}(t)$. Then

$$
A_{N}(t)=\sum_{|I|=N} t^{I} A_{I}
$$

where $\left.\sum_{j=1}^{n} \mathbb{H}\left(\mu_{j}\right\lrcorner \eta_{\left(i_{1}, \ldots, i_{j}-1, \ldots, i_{n}\right)}^{\alpha}\right)=A_{I},{ }_{\beta}^{\alpha} \bar{\theta}_{p}^{\beta}$. In particular, $\left.\mathbb{M}\left(\mu_{i}\right\lrcorner \theta_{p}^{\alpha}\right)=A_{i},{ }_{\beta}^{\alpha} \bar{\theta}_{p}^{\beta}$.

Detailed discussion of homogeneous parts of $A(t)$ is given as follows, and will be useful in the computation.

(1) The first two homogeneous parts $A_{1}(t)$ and $A_{2}(t)$ :

$$
\begin{gathered}
\left.\mathbb{H}\left(\mu_{i}\right\lrcorner \theta_{p}^{\alpha}\right)=A_{i},{ }_{\beta}^{\alpha} \bar{\theta}_{p}^{\beta} \quad \text { for }|I|=1, \\
\begin{cases}\left.\left.\mathbb{H}\left(\mu_{i}\right\lrcorner \eta_{j}^{\alpha}\right)+\mathbb{U}\left(\mu_{j}\right\lrcorner \eta_{i}^{\alpha}\right)=A_{\left(0, \ldots, 0,1_{i-\mathrm{th}}, 0, \ldots, 0, \underline{1}_{j t h}, 0, \ldots, 0\right),{ }_{\beta}^{\alpha} \bar{\theta}_{p}^{\beta}} & \text { for }|I|=2, i \neq j, \\
\left.\mathbb{U}\left(\mu_{i}\right\lrcorner \eta_{i}^{\alpha}\right)=A_{\left(0, \ldots, 0, \underline{2}_{i-\mathrm{th}}, 0, \ldots, 0\right),{ }_{\beta} \bar{\theta}_{p}^{\beta}} & \text { for }|I|=2, i=j .\end{cases}
\end{gathered}
$$

Set

$$
A_{i j}:= \begin{cases}A_{\left(0, \ldots, 0, \underline{1}_{i-\mathrm{th}}, 0, \ldots, 0, \underline{1}_{j t h}, 0, \ldots, 0\right)} & \text { for } i<j \\ 2 A_{\left(0, \ldots, 0, \underline{2}_{i-\mathrm{h}}, 0, \ldots, 0\right)} & \text { for } i=j \\ A_{j i} & \text { for } i>j .\end{cases}
$$

Then it is easy to check that

$$
\sum_{i, j=1}^{n} t_{i} t_{j} A_{i j}=2 \sum_{|I|=2} t^{I} A_{I} \quad \text { and } \quad \frac{\partial}{\partial t_{i}} \frac{\partial}{\partial t_{j}} A_{2}(t)=A_{i j} .
$$

(2) The general homogeneous part $A_{N}(t)$ : 
Define $A_{i_{1}, \ldots, i_{N}}$, symmetric in all its subscripts, such that

$$
\sum_{i_{1}, \ldots, i_{N}=1}^{n} t_{i_{1}} \ldots t_{i_{N}} A_{i_{1}, \ldots, i_{N}}=N ! A_{N}(t) .
$$

From this, we can deduce that

$$
\frac{N !}{j_{1} ! j_{2} ! \ldots j_{n} !} A_{i_{1}, \ldots, i_{N}}=N ! A_{\left(j_{1}, j_{2}, \ldots, j_{n}\right)}
$$

if $k$ appears $j_{k}$ times in $i_{1}, \ldots, i_{N}$. Here $\sum_{k=1}^{n} j_{k}=N$ since we are considering the homogeneous $N$-part. Also it is easy to check that

$$
\frac{\partial}{\partial t_{i_{1}}} \ldots \frac{\partial}{\partial t_{i_{N}}} A_{N}(t)=A_{i_{1}, \ldots, i_{N}} .
$$

Set

$$
\Theta_{p}(t)=\left(\begin{array}{c}
\theta_{p}^{1}(t) \\
\vdots \\
\theta_{p}^{g}(t)
\end{array}\right) \quad \text { and } \quad \Theta_{p}=\left(\begin{array}{c}
\theta_{p}^{1} \\
\vdots \\
\theta_{p}^{g}
\end{array}\right) .
$$

Thus using $A(t)$ and $E(t)$, we rewrite (2-1) as

$$
\Theta_{p}(t)=\left(\mathbb{1}_{g} A(t)\right)\left(\begin{array}{c}
\Theta_{p} \\
\bar{\Theta}_{p}
\end{array}\right)+E(t) .
$$

Since a holomorphic one-form on a Riemann surface is uniquely determined by its integration on $A$ cycles, it is clear that $\left\{\theta_{p}^{\alpha}(t)\right\}_{\alpha=1}^{g}$ being a frame of $H^{0}\left(X_{t}, \Omega_{X_{t}}^{1}\right)$ on $X_{t}$ is equivalent to nondegeneracy of the $A$ period matrix $\sigma_{\alpha \beta}(t)$ on $X_{t}$, i.e.,

$$
\operatorname{det}\left(\sigma_{\alpha \beta}(t)\right)=\operatorname{det}\left(\int_{A_{\alpha}} \theta_{p}^{\beta}(t)\right) \neq 0 \Longleftrightarrow \operatorname{det}\left(\mathbb{1}_{g}+A(t)^{T}\right) \neq 0,
$$

where $A(t)^{T}$ is the transpose of $A(t)$. And when $\left\{\theta_{p}^{\alpha}(t)\right\}_{\alpha=1}^{g}$ becomes a frame, we have the Hodge-Riemann bilinear relations on $X_{t}$

$$
\left\{\begin{array}{l}
0=\frac{\mathrm{i}}{2} \int_{X_{t}} \theta_{p}^{\alpha}(t) \wedge \theta_{p}^{\beta}(t), \\
0<\frac{\mathrm{i}}{2} \int_{X_{t}} \theta_{p}^{\alpha}(t) \wedge \bar{\theta}_{p}^{\beta}(t),
\end{array}\right.
$$

which, together with (2-2), implies that

$$
\left\{\begin{array}{l}
0=\frac{\mathrm{i}}{2} \int_{X_{p}}\left(\theta_{p}^{\alpha}+A(t)_{\gamma}^{\alpha} \bar{\theta}^{\gamma}+E^{\alpha}(t)\right) \wedge\left(\theta_{p}^{\beta}+A(t)_{\lambda}^{\beta} \bar{\theta}^{\lambda}+E^{\beta}(t)\right), \\
0<\frac{\mathrm{i}}{2} \int_{X_{p}}\left(\theta_{p}^{\alpha}+A(t)_{\gamma}^{\alpha} \bar{\theta}_{p}^{\gamma}+E^{\alpha}(t)\right) \wedge\left(\bar{\theta}_{p}^{\beta}+\overline{A(t)}_{\lambda}^{\beta} \theta_{p}^{\lambda}+\bar{E}^{\beta}(t)\right) .
\end{array}\right.
$$


Thus, by type consideration and Stokes's theorem, we have

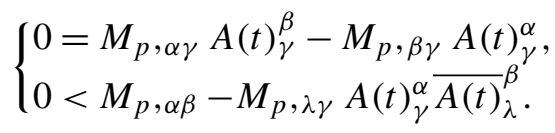

The matrix forms of these are given by

$$
\left\{\begin{array}{l}
A(t) M_{p}=\left(A(t) M_{p}\right)^{T}, \\
M_{p}-A(t) M_{p} \overline{A(t)}^{T}>0 .
\end{array}\right.
$$

As our deformation formula is local, $\left\{\theta_{p}^{\alpha}(t)\right\}_{\alpha=1}^{g}$ is always a frame, as $t \in \Delta_{p, \epsilon}$ with $\epsilon$ sufficiently small. Therefore, (2-3) and (2-4) hold.

On our Kuranishi coordinate $\Delta_{p, \epsilon}$, the period map $\Pi: \mathscr{T}_{g} \rightarrow \mathscr{H}_{g}$ can be written out quite explicitly:

$$
\begin{aligned}
& \Pi(t)_{\alpha \beta}=\int_{B_{\alpha}} \sigma(t)^{\gamma \beta} \theta_{p}^{\gamma}(t)=\int_{B_{\alpha}} \sigma(t)^{\gamma \beta}\left(\theta_{p}^{\gamma}+A(t),{ }_{\delta}^{\gamma} \bar{\theta}_{p}^{\delta}\right) \\
& =\pi_{p, \alpha \gamma} \sigma(t)^{\gamma \beta}+\bar{\pi}_{p}, \alpha \delta A(t)_{\delta}^{\gamma} \sigma(t)^{\gamma \beta},
\end{aligned}
$$

where $\sigma(t)^{\alpha \beta}$ is the inverse matrix of $\sigma(t)_{\alpha \beta}$. Here $\sigma(t)_{\alpha \beta}$ is given by

$$
\sigma_{\alpha \beta}(t)=\int_{A_{\alpha}} \theta_{p}^{\beta}(t)=\left(\mathbb{1}_{g}+A(t)^{T}\right)_{\alpha \beta}
$$

By (2-6), we formulate (2-5) into the matrix type to get

$$
\Pi(t)=\left(\pi_{p}+\bar{\pi}_{p} A(t)^{T}\right)\left(\mathbb{1}_{g}+A(t)^{T}\right)^{-1} .
$$

Full expansion of the period map. We are going to give (2-7) a full expansion, writing out every order part explicitly.

Lemma 2.3 [Farkas and Kra 1992, Proposition III.2.3]. If $\phi$ and $\psi$ are two d-closed one-forms on a Riemann surface $X$, then

$$
\int_{X} \phi \wedge \psi=\sum_{\gamma}\left(\int_{A_{\gamma}} \phi \int_{B_{\gamma}} \psi-\int_{B_{\gamma}} \phi \int_{A_{\gamma}} \psi\right),
$$

where $\left\{A_{\gamma}, B_{\gamma}\right\}_{\gamma=1}^{g}$ is the symplectic basis of $X$.

Lemma 2.4. We hve

$$
\left.\left.\int_{A_{\alpha}} \mathbb{U}\left(\mu_{k}\right\lrcorner \theta^{\beta}\right)=\frac{\mathrm{i}}{2} M^{\alpha \gamma} \int_{X} \theta^{\gamma} \wedge \mathbb{M}\left(\mu_{k}\right\lrcorner \theta^{\beta}\right),
$$

where $\left\{\theta^{\alpha}\right\}_{\alpha=1}^{g}$ is the canonical basis of holomorphic one-forms on $X$ and $M^{\alpha \beta}$ is the inverse matrix of $M_{\alpha \beta}=\operatorname{Im}\left(\pi_{\alpha \beta}\right)$. 


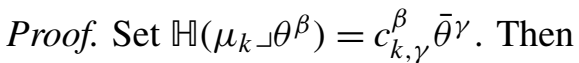

$$
\left.\int_{A_{\alpha}} \mathbb{H}\left(\mu_{k}\right\lrcorner \theta^{\beta}\right)=c_{k, \alpha}^{\beta},
$$

while Lemma 2.3 implies that

$$
\left.\mathrm{i} \int_{X_{0}} \theta^{\gamma} \wedge \mathbb{M}\left(\mu_{k}\right\lrcorner \theta^{\beta}\right)=\mathrm{i} c_{k, \gamma}^{\beta} \int_{X_{0}} \theta^{\alpha} \wedge \bar{\theta}^{\gamma}=2 c_{k, \gamma}^{\beta} M_{\alpha \gamma} .
$$

Finally we have the equality above.

Theorem 2.5. The period map $\Pi: \mathscr{T}_{g} \rightarrow \mathscr{H}_{g}$ has the full expansion on the Kuranishi coordinate $\Delta_{p, \epsilon}$

$$
\begin{aligned}
\left.\left.\Pi_{\alpha \beta}(t)=\Pi_{\alpha \beta}(0)+\int_{X_{0}} \theta^{\alpha} \wedge \mathbb{M}(\mu(t)\lrcorner \theta^{\beta}\right)+\int_{X_{0}} \theta^{\alpha} \wedge \mathbb{M}(\mu(t)\lrcorner \eta_{t, 1}^{\beta}\right) \\
\left.\left.\quad-\frac{\mathrm{i}}{2} \int_{X_{0}} \theta^{\alpha} \wedge \mathbb{H}(\mu(t)\lrcorner \theta^{\delta}\right) M^{\delta \gamma} \int_{X_{0}} \theta^{\gamma} \wedge \mathbb{M}(\mu(t)\lrcorner \theta^{\beta}\right) \\
+\sum_{k \geq 3} \sum_{\substack{m_{i}>0,1 \leq i \leq l \\
m_{1}+\cdots+m_{l}=k}}\left\{(-1)^{l-1} \int_{X_{0}} \theta^{\alpha} \wedge \mathbb{H}(\mu(t)\lrcorner \eta_{t, m_{1}-1}^{\alpha_{1}}\right) \\
\left.\times \frac{\mathrm{i}}{2} M^{\alpha_{1} \alpha_{2}} \int_{X_{0}} \theta^{\alpha_{2}} \wedge \mathbb{M}(\mu(t)\lrcorner \eta_{t, m_{2}-1}^{\alpha_{3}}\right) \cdots \\
\left.\left.\times \frac{\mathrm{i}}{2} M^{\alpha_{2 l-3} \alpha_{2 l-2}} \int_{X_{0}} \theta^{\alpha_{2 l-2}} \wedge \mathbb{M}(\mu(t)\lrcorner \eta_{t, m_{l}-1}^{\beta}\right)\right\},
\end{aligned}
$$

where $\eta_{t, N}^{\alpha}$ is the $N$-th order part of the expansion of $\eta_{t}^{\alpha}$ from the deformation formula of $\theta^{\alpha}$ by Theorem 2.1 and $M^{\alpha \beta}$ is the inverse matrix of $M_{p}=\operatorname{Im}\left(\pi_{p}\right)$.

Proof. Write out $A, B$ periods of $X_{t}$ as $\sigma_{\alpha \beta}(t), \pi_{\alpha \beta}(t)$, respectively. Then

$$
\begin{aligned}
& \left.\sigma_{\alpha \beta}(t)=\int_{A_{\alpha}} \theta^{\beta}(t)=\int_{A_{\alpha}}\left(\theta^{\beta}+\sum_{|I| \geq 1} t^{I}\left(\sum_{j=1}^{n} \mathbb{M}\left(\mu_{j}\right\lrcorner \eta_{\left(i_{1}, \ldots, i_{j}-1, \ldots, i_{n}\right)}^{\beta}\right)\right)\right), \\
& \pi_{\alpha \beta}(t)=\int_{B_{\alpha}} \theta^{\beta}(t)=\int_{B_{\alpha}}\left(\theta^{\beta}+\sum_{|I| \geq 1} t^{I}\left(\sum_{j=1}^{n} \mathbb{M}\left(\mu_{j}\right\lrcorner \eta_{\left(i_{1}, \ldots, i_{j}-1, \ldots, i_{n}\right)}^{\beta}\right)\right) .
\end{aligned}
$$

Those expansion coefficients are

$$
\left\{\begin{array}{l}
\left.\sigma_{\alpha \beta, I}=\int_{A_{\alpha}} \sum_{j=1}^{n} \mathbb{H}\left(\mu_{j}\right\lrcorner \eta_{\left(i_{1}, \ldots, i_{j}-1, \ldots, i_{n}\right)}^{\beta}\right), \\
\left.\pi_{\alpha \beta, I}=\int_{B_{\alpha}} \sum_{j=1}^{n} \mathbb{M}\left(\mu_{j}\right\lrcorner \eta_{\left(i_{1}, \ldots, i_{j}-1, \ldots, i_{n}\right)}^{\beta}\right) .
\end{array}\right.
$$

Thus the period map can be computed as 


$$
\Pi_{\alpha \beta}(t)=\int_{B_{\alpha}} \tilde{\theta}_{t}^{\beta}=\int_{B_{\alpha}} \sigma^{\gamma \beta}(t) \theta_{t}^{\gamma}=\sigma^{\gamma \beta}(t) \pi_{\alpha \gamma}(t),
$$

where $\tilde{\theta}^{\beta}(t)$ is the canonical basis of holomorphic one-forms on $X_{t}$ and $\sigma^{\beta \alpha}(t)$ is the inverse matrix of $\sigma_{\alpha \beta}(t)$.

Now we only focus on the expansion of $\sigma^{\gamma \beta}(t)$, since the expansion of $\pi_{\alpha \beta}(t)$ is already obtained. Set

$$
\sigma^{\alpha \beta}(t)=\delta_{\alpha \beta}+\sum_{|I| \geq 1} t^{I} \sigma_{I}^{\alpha \beta}
$$

then

$$
\begin{gathered}
\delta_{\alpha \gamma}=\sigma_{\alpha \beta}(t) \sigma^{\beta \gamma}(t)=\left(\delta_{\alpha \beta}+\sum_{|I| \geq 1} t^{I} \sigma_{\alpha \beta, I}\right)\left(\delta_{\beta \gamma}+\sum_{|I| \geq 1} t^{I} \sigma_{I}^{\beta \gamma}\right) \\
=\delta_{\alpha \gamma}+t^{I} \sigma_{\alpha \gamma, I}+t^{I} \sigma_{I}^{\alpha \gamma}+t^{I+J} \sigma_{\alpha \beta, I} b_{J}^{\beta \gamma} \\
=\delta_{\alpha \gamma}+\sum_{i=1}^{n} t_{i}\left(\sigma_{\alpha \gamma,\left(0, \ldots,{ }_{i-\mathrm{th}}^{1}, \ldots, 0\right)}+\sigma_{\substack{\left(0, \ldots, i_{i-\mathrm{th}}, \ldots, 0\right) \\
\alpha \gamma}}\right. \\
\quad+\sum_{|K| \geq 2} t^{K}\left(\sigma_{\alpha \gamma, K}+\sigma_{K}^{\alpha \gamma}+\sum_{\substack{|I| \geq 1,|J| \geq 1 \\
I+J=K}} \sigma_{\alpha \beta, I} \sigma_{J}^{\beta \gamma}\right) .
\end{gathered}
$$

Compare both sides of this equation to get

$$
\left\{\begin{array}{l}
\sigma_{\alpha \gamma,\left(0, \ldots, \underline{1}_{i-\mathrm{h}}, \ldots, 0\right)}+\sigma_{(0, \ldots, 1, \ldots, 0)}^{\alpha \gamma}=0, \\
\sigma_{K}^{\alpha \gamma}+\sigma_{\alpha \gamma, K}+\sum_{\substack{i-\mathrm{th} \\
|I| \geq 1,|J| \geq 1 \\
I+J=K}} \sigma_{\alpha \beta, I} \sigma_{J}^{\beta \gamma}=0 .
\end{array}\right.
$$

Define the homogeneous parts of $\sigma_{\alpha \beta}(t), \pi_{\alpha \beta}(t)$ and $\sigma^{\alpha \beta}(t)$ as

$$
\left(\sigma_{\alpha \beta}\right)_{k}:=\sum_{|K|=k} t^{K} \sigma_{\alpha \beta, K}, \quad\left(\pi_{\alpha \beta}\right)_{k}:=\sum_{|K|=k} t^{K} \pi_{\alpha \beta, K}, \quad\left(\sigma^{\alpha \beta}\right)_{k}:=\sum_{|K|=k} t^{K} \sigma_{K}^{\alpha \beta}
$$

Using these definitions, we rewrite (2-10) to obtain the recursive relation

$$
\left\{\begin{array}{l}
\left(\sigma_{\alpha \gamma}\right)_{1}+\left(\sigma^{\alpha \gamma}\right)_{1}=0, \\
\left(\sigma_{\alpha \gamma}\right)_{k}+\left(\sigma^{\alpha \gamma}\right)_{k}+\sum_{\substack{i \geq 1, j \geq 1 \\
i+j=k}}\left(\sigma_{\alpha \beta}\right)_{i}\left(\sigma^{\beta \gamma}\right)_{j}=0 .
\end{array}\right.
$$

From (2-9) and (2-11), we get 


$$
\begin{aligned}
\Pi_{\alpha \beta}(t)= & \sigma^{\gamma \beta}(t) \pi_{\alpha \gamma}(t)=\left(\delta_{\gamma \beta}+\sum_{|I| \geq 1} t^{I} \sigma_{I}^{\gamma \beta}\right)\left(\pi_{\alpha \gamma}(0)+\sum_{|I| \geq 1} t^{I} \pi_{\alpha \gamma, I}\right) \\
= & \pi_{\alpha \beta}(0)+\sum_{i=1}^{n} t_{i}\left(\pi_{\alpha \gamma}(0) \sigma_{\left(0, \ldots, \underline{1}_{i-\mathrm{th}}, \ldots, 0\right)}^{\gamma \beta}+\pi_{\alpha \beta,\left(0, \ldots, \underline{1}_{i-\mathrm{th}}, \ldots, 0\right)}\right) \\
& +\sum_{|K| \geq 2} t^{K}\left\{\pi_{\alpha \beta, K}+\pi_{\alpha \gamma}(0) \sigma_{K}^{\gamma \beta}+\sum_{\substack{|I| \geq 1,|J| \geq 1 \\
I+J=K}} \pi_{\alpha \gamma, I} \sigma_{J}^{\gamma \beta}\right\} \\
= & \Pi_{\alpha \beta}(0)+\pi_{\alpha \gamma}(0)\left(\sigma^{\gamma \beta}\right)_{1}+\left(\pi_{\alpha \beta}\right)_{1} \sum_{k \geq 2}\left\{\left(\pi_{\alpha \beta}\right)_{k}+\pi_{\alpha \gamma}(0)\left(\sigma^{\gamma \beta}\right)_{k}+\sum_{\substack{i \geq 1, j \geq 1 \\
i+j=k}}\left(\pi_{\alpha \gamma}\right)_{i}\left(\sigma^{\gamma \beta}\right)_{j}\right\} .
\end{aligned}
$$

After observing the formula above, we need to use the recursion relation (2-12) to get the full expansion of $\sigma^{\alpha \beta}(t)$.

Claim. $\sigma^{\alpha \beta}(t)$ has the expansion

$$
\begin{aligned}
\sigma^{\alpha \beta}(t)= & \left.\delta_{\alpha \beta}-\int_{A_{\alpha}} \mathbb{H}(\mu(t)\lrcorner \theta^{\beta}\right) \\
& \left.+\sum_{k \geq 2} \sum_{\substack{m_{i}>0,1 \leq i \leq n \\
m_{1}+\cdots+m_{l}=k}}\left\{(-1)^{l} \int_{A_{\alpha}} \mathbb{H}(\mu(t)\lrcorner \eta_{t, m_{1}-1}^{\alpha_{1}}\right) \int_{A_{\alpha_{1}}} \mathbb{H}(\mu(t)\lrcorner \eta_{t, m_{2}-1}^{\alpha_{2}}\right) \cdots \\
& \left.\left.\left.\times \int_{A_{\alpha_{l-2}}} \mathbb{H}(\mu(t)\lrcorner \eta_{t, m_{l-1}-1}^{\alpha_{l-1}}\right) \int_{A_{\alpha_{l-1}}} \mathbb{H}(\mu(t)\lrcorner \eta_{t, m_{l}-1}^{\beta}\right)\right\} .
\end{aligned}
$$

Proof. Use an induction argument: For $k=1$, according to (2-12), the first-order part of $\sigma^{\alpha \beta}(t)$ is given by

$$
\left.\left(\sigma^{\alpha \beta}\right)_{1}=-\left(\sigma_{\alpha \beta}\right)_{1}=-\int_{A_{\alpha}} \nVdash(\mu(t)\lrcorner \theta^{\beta}\right) .
$$

Assume that the homogeneous parts with orders less than or equal to $k-1$ are given by the formula in the claim. Then the $k$-th term is

$$
\begin{aligned}
\left(\sigma^{\alpha \beta}\right)_{k}= & -\left(\sigma_{\alpha \beta}\right)_{k}-\sum_{\substack{i \geq 1, j \geq 1 \\
i+j=k}}\left(\sigma_{\alpha \gamma}\right)_{i}\left(\sigma^{\gamma \beta}\right)_{j} \\
= & \left.\left.-\int_{A_{\alpha}} \mathbb{H}(\mu(t)\lrcorner \eta_{t, k-1}^{\beta}\right)-\sum_{\substack{i \geq 1, j \geq 1 \\
i+j=k}} \int_{A_{\alpha}} \mathbb{H}(\mu(t)\lrcorner \eta_{t, i-1}^{\gamma}\right) \\
& \left.\left.\times\left\{\sum_{\substack{m_{i}>0,1 \leq i \leq l \\
m_{1}+\cdots+m_{l}=j}}(-1)^{l} \int_{A_{\gamma}} \mathbb{H}(\mu(t)\lrcorner \eta_{t, m_{1}-1}^{\alpha_{1}}\right) \cdots \int_{A_{\alpha_{l-1}}} \mathbb{H}(\mu(t)\lrcorner \eta_{t, m_{l}-1}^{\beta}\right)\right\}
\end{aligned}
$$




$$
\begin{aligned}
& \left.\left.=-\int_{A_{\alpha}} \mathbb{U}(\mu(t)\lrcorner \eta_{t, k-1}^{\beta}\right)+\sum_{\substack{i \neq k, m_{j}>0,1 \leq j \leq l \\
i+m_{1}+\cdots+m_{l}=k}}(-1)^{l+1} \int_{A_{\alpha}} \mathbb{H}(\mu(t)\lrcorner \eta_{t, i-1}^{\gamma}\right) \\
& \left.\left.\times \int_{A_{\gamma}} \mathbb{H}(\mu(t)\lrcorner \eta_{t, m_{1}-1}^{\alpha_{1}}\right) \cdots \int_{A_{\alpha_{l}-1}} \mathbb{H}(\mu(t)\lrcorner \eta_{t, m_{l}-1}^{\beta}\right) \\
& \left.\left.=-\int_{A_{\alpha}} \mathbb{H}(\mu(t)\lrcorner \eta_{t, k-1}^{\beta}\right)+\sum_{\substack{m_{1} \neq k, m_{i}>0,2 \leq i \leq l \\
m_{1}+\cdots+m_{l}=k}}(-1)^{l} \int_{A_{\alpha}} \mathbb{U}(\mu(t)\lrcorner \eta_{t, m_{1}-1}^{\alpha_{1}}\right) \\
& \left.\left.\times \int_{A_{\alpha_{1}}} \mathbb{H}(\mu(t)\lrcorner \eta_{t, m_{1}-1}^{\alpha_{2}}\right) \cdots \int_{A_{\alpha_{l-1}}} \mathbb{U}(\mu(t)\lrcorner \eta_{t, m_{l}-1}^{\beta}\right) \\
& \left.=\sum_{\substack{m_{i}>0,1 \leq i \leq l \\
m_{1}+\cdots+m_{l}=k}}(-1)^{l} \int_{A_{\alpha}} \mathbb{M}(\mu(t)\lrcorner \eta_{t, m_{1}-1}^{\alpha_{1}}\right) \\
& \left.\left.\times \int_{A_{\alpha_{1}}} \mathbb{U}(\mu(t)\lrcorner \eta_{t, m_{1}-1}^{\alpha_{2}}\right) \cdots \int_{A_{\alpha_{l}-1}} \mathbb{M}(\mu(t)\lrcorner \eta_{t, m_{l}-1}^{\beta}\right) .
\end{aligned}
$$

Thus our claim has been proved.

Let us proceed to the expansion of $\Pi_{\alpha \beta}(t)$. Use the claim and the expansion formula of $\Pi_{\alpha \beta}(t)$ above to get

$$
\begin{aligned}
& \Pi_{\alpha \beta}(t) \\
& \left.=\Pi_{\alpha \beta}(0)+\pi_{\alpha \gamma}(0)\left(\sigma^{\gamma \beta}\right)_{1}+\int_{B_{\alpha}} \mathbb{H}(\mu(t)\lrcorner \theta^{\beta}\right) \\
& \left.\left.+\sum_{k \geq 2}\left\{\int_{B_{\alpha}} \mathbb{M}(\mu(t)\lrcorner \eta_{t, k-1}^{\beta}\right)+\pi_{\alpha \gamma}(0)\left(\sigma^{\gamma \beta}\right)_{k}+\sum_{\substack{i \geq 1, j \geq 1 \\
i+j=k}} \int_{B_{\alpha}} \mathbb{U}(\mu(t)\lrcorner \eta_{t, i-1}^{\gamma}\right)\left(\sigma^{\gamma \beta}\right)_{j}\right\} \\
& \left.\left.=\Pi_{\alpha \beta}(0)-\pi_{\alpha \gamma}(0) \int_{A_{\gamma}} \llbracket(\mu(t)\lrcorner \theta^{\beta}\right)+\int_{B_{\alpha}} \mathbb{M}(\mu(t)\lrcorner \theta^{\beta}\right) \\
& \left.+\sum_{k \geq 2}\left\{\int_{B_{\alpha}} \mathbb{M}(\mu(t)\lrcorner \eta_{t, k-1}^{\beta}\right)-\pi_{\alpha \gamma}(0) \int_{A_{\gamma}} \mathbb{M}(\mu(t)\lrcorner \eta_{t, k-1}^{\beta}\right) \\
& \left.\left.\left.-\sum_{\substack{i \geq 1, j \geq 1 \\
i+j=k}} \pi_{\alpha \gamma}(0) \int_{A_{\gamma}} \mathbb{H}(\mu(t)\lrcorner \eta_{t, i-1}^{\sigma}\right)\left(\sigma^{\sigma \beta}\right)_{j}+\sum_{\substack{i \geq 1, j \geq 1 \\
i+j=k}} \int_{B_{\alpha}} \mathbb{H}(\mu(t)\lrcorner \eta_{t, i-1}^{\sigma}\right)\left(\sigma^{\sigma \beta}\right)_{j}\right\} \\
& \left.=\Pi_{\alpha \beta}(0)+\int_{X_{0}} \theta^{\alpha} \wedge \mathfrak{M}(\mu(t)\lrcorner \theta^{\beta}\right) \\
& \left.\left.+\sum_{k \geq 2}\left\{\int_{X_{0}} \theta^{\alpha} \wedge \mathbb{M}(\mu(t)\lrcorner \eta_{t, k-1}^{\beta}\right)+\sum_{\substack{i \geq 1, j \geq 1 \\
i+j=k}} \int_{X_{0}} \theta^{\alpha} \wedge \mathbb{H}(\mu(t)\lrcorner \eta_{t, i-1}^{\sigma}\right)\left(\sigma^{\sigma \beta}\right)_{j}\right\}
\end{aligned}
$$




$$
\begin{aligned}
& \left.=\Pi_{\alpha \beta}(0)+\int_{X_{0}} \theta^{\alpha} \wedge \Vdash(\mu(t)\lrcorner \theta^{\beta}\right) \\
& \left.+\sum_{k \geq 2}\left\{\int_{X_{0}} \theta^{\alpha} \wedge \mathbb{M}(\mu(t)\lrcorner \eta_{t, k-1}^{\beta}\right)+\sum_{\substack{i \geq 1, j \geq 1 \\
i+j=k}} \int_{X_{0}} \theta^{\alpha} \wedge \mathbb{M}(\mu(t)\lrcorner \eta_{t, i-1}^{\sigma}\right) \\
& \left.\left.\left.\times\left[\sum_{\substack{m_{i}>0,1 \leq i \leq l \\
m_{1}+\cdots+m_{l}=j}}(-1)^{l} \int_{A_{\sigma}} \mathbb{H}(\mu(t)\lrcorner \eta_{t, m_{1}-1}^{\alpha_{1}}\right) \cdots \int_{A_{\alpha_{l-1}}} \mathbb{H}(\mu(t)\lrcorner \eta_{t, m_{l}-1}^{\beta}\right)\right]\right\} \\
& \left.=\Pi_{\alpha \beta}(0)+\int_{X_{0}} \theta^{\alpha} \wedge \mathfrak{M}(\mu(t)\lrcorner \theta^{\beta}\right) \\
& \left.+\sum_{k \geq 2}\left\{\int_{X_{0}} \theta^{\alpha} \wedge \mathbb{H}(\mu(t)\lrcorner \eta_{t, k-1}^{\beta}\right)+\sum_{\substack{i \neq k, m_{j}>0,1 \leq j \leq l \\
i+m_{1}+\cdots+m_{l}=k}}(-1)^{l} \int_{X_{0}} \theta^{\alpha} \wedge \mathbb{H}(\mu(t)\lrcorner \eta_{t, i-1}^{\sigma}\right) \\
& \left.\left.\left.\times \int_{A_{\sigma}} \mathbb{H}(\mu(t)\lrcorner \eta_{t, m_{1}-1}^{\alpha_{1}}\right) \cdots \int_{A_{\alpha_{l-1}}} \mathbb{H}(\mu(t)\lrcorner \eta_{t, m_{l}-1}^{\beta}\right)\right\} \\
& \left.=\Pi_{\alpha \beta}(0)+\int_{X_{0}} \theta^{\alpha} \wedge \mathbb{M}(\mu(t)\lrcorner \theta^{\beta}\right) \\
& +\sum_{k \geq 2} \sum_{\substack{m_{i}>0,1 \leq i \leq l \\
m_{1}+\cdots+m_{l}=k}}\left\{(-1)^{l-1} \int_{X_{0}} \theta^{\alpha} \wedge \mathbb{M}(\mu(t)\lrcorner \eta_{t, m_{1}-1}^{\alpha_{1}}\right) \times \\
& \left.\left.\left.\frac{\mathrm{i}}{2} M^{\alpha_{1} \alpha_{2}} \int_{X_{0}} \theta^{\alpha_{2}} \wedge \mathbb{M}(\mu(t)\lrcorner \eta_{t, m_{2}-1}^{\alpha_{3}}\right) \cdots \frac{\mathrm{i}}{2} M^{\alpha_{2 l-3} \alpha_{2 l-2}} \int_{X_{0}} \theta^{\alpha_{2 l-2}} \wedge \mathbb{M}(\mu(t)\lrcorner \eta_{t, m_{l}-1}^{\beta}\right)\right\}
\end{aligned}
$$

and this concludes the proof of Theorem 2.5.

Corollary 2.6. For every $N \geq 0$,

$$
\left.\int_{X_{0}} \theta^{\alpha} \wedge \mathbb{M}(\mu(t)\lrcorner \eta_{t, N}^{\beta}\right)
$$

is a symmetric matrix of $(\alpha, \beta)$.

Proof. We again use an induction argument.

When $\left.N=0, \int_{X_{0}} \theta^{\alpha} \wedge \mathbb{H}(\mu(t)\lrcorner \theta^{\beta}\right)$ is the homogeneous part of first order of $\Pi_{\alpha \beta}(t)$. It is easy to see that $\Pi_{\alpha \beta}(t)$ is a symmetric matrix of $(\alpha, \beta)$, and thus the homogeneous part of every order of its expansion will be symmetric in $(\alpha, \beta)$, and in particular the first order.

Assume that $\left.\int_{X_{0}} \theta^{\alpha} \wedge \mathbb{U}(\mu(t)\lrcorner \eta_{t, N}^{\beta}\right)$, with $N \leq k-1$, is symmetric in $(\alpha, \beta)$. Now we come to the case $N=k$. By Theorem 2.5, the $(k+1)$-th homogeneous part of the expansion of $\Pi_{\alpha \beta}$ is 


$$
\begin{aligned}
\left.\int_{X_{0}} \theta^{\alpha} \wedge \mathbb{H}(\mu(t)\lrcorner \eta_{t, k}^{\beta}\right)+\sum_{\begin{array}{c}
m_{i} \leq k \\
m_{1}+m_{2}+\cdots+m_{l}=k+1
\end{array}} & \left.(-1)^{l-1} \int_{X_{0}} \theta^{\alpha} \wedge \mathbb{H}(\mu(t)\lrcorner \eta_{t, m_{1}-1}^{\alpha_{1}}\right) \\
\times & \left.\frac{\mathrm{i}}{2} M^{\alpha_{1} \alpha_{2}} \int_{X_{0}} \theta^{\alpha_{2}} \wedge \mathbb{H}(\mu(t)\lrcorner \eta_{t, m_{2}-1}^{\alpha_{3}}\right) \cdots \\
& \left.\times \frac{\mathrm{i}}{2} M^{\alpha_{2 l-3} \alpha_{2 l-2}} \int_{X_{0}} \theta^{\alpha_{2 l-2}} \wedge \mathbb{H}(\mu(t)\lrcorner \eta_{t, m_{l}-1}^{\beta}\right)
\end{aligned}
$$

and is thus symmetric in $(\alpha, \beta)$. By use of the induction assumption and the symmetric matrix $M^{\alpha \beta}$, the second summand of the above formula is symmetric in $(\alpha, \beta)$. Thus $\left.\int_{X_{0}} \theta^{\alpha} \wedge \mathbb{M}(\mu(t)\lrcorner \eta_{t, k}^{\beta}\right)$ is symmetric in $(\alpha, \beta)$.

Remark 2.7. It is easy to see that $\left.\frac{\mathrm{i}}{2} \int_{X_{0}} \theta^{\alpha} \wedge \mathbb{M}(\mu(t)\lrcorner \eta_{t, N}^{\beta}\right)=M_{\alpha \gamma} A_{N+1}(t)_{\gamma}^{\beta}$. Thus $M A_{N+1}^{T}(t)$ is a symmetric matrix for $N \geq 0$.

\section{A global result on deformation of holomorphic one-forms}

This section will present a global convergence of the deformation of holomorphic one-forms in $L^{2}$ norm as a result of the following quasi-isometry for the operator $\bar{\partial}^{*} \circ \mathbb{G} \circ \partial$.

Proposition 3.1 [Liu et al. 2012a, Theorem 2.2.(3)]. Let $T^{p, q}=\partial T^{p-1, q} \in A^{p, q}(M)$ on a compact Kähler manifold $M$. Then we have the inequality

$$
\left\|\bar{\partial}^{*} \circ \mathbb{G} \circ \partial T^{p-1, q}\right\|_{L^{2}} \leq\left\|T^{p-1, q}\right\|_{L^{2}} .
$$

Furthermore, if $T^{p-1, q}$ is $\partial^{*}$-exact, then the equality in (3-1) holds, i.e.,

$$
\left\|\bar{\partial}^{*} \circ \mathbb{G} \circ \partial T^{p-1, q}\right\|_{L^{2}}=\left\|T^{p-1, q}\right\|_{L^{2}} .
$$

This proposition was originally proved by step-by-step spectral decompositions in the preliminary version of [Liu et al. 2012a]. It is motivated by an attempt to prove the global Torelli theorem for the Teichmüller space of CY manifolds and inspired by the integral operators $P$ and $T$ defined by

$$
\begin{array}{ll}
\operatorname{Ph}(\zeta)=-\frac{1}{\pi} \iint_{\mathbb{C}} h(z)\left(\frac{1}{z-\zeta}-\frac{1}{z}\right) d x d y, & \text { for functions } h \in L^{p}, p>2, \\
T h(\zeta)=\lim _{\varepsilon \rightarrow 0}-\frac{1}{\pi} \iint_{|z-\zeta|>\varepsilon} \frac{h(z)}{(z-\zeta)^{2}} d x d y, \quad \text { for functions } h \in C_{0}^{2} .
\end{array}
$$

These integral operators satisfy $(P h)_{\bar{z}}=h,(P h)_{z}=T h$, and the (quasi-)isometry relations

$\|T h\|_{L^{2}}=\|h\|_{L^{2}},\|T h\|_{L^{p}} \leq C_{p}\|h\|_{L^{p}}$, for any $p>1$, with $C_{p} \rightarrow 1$ for $p \rightarrow 2$, 
which all appear in the classical Teichmüller theory for Riemann surfaces; see [Ahlfors 1966], whose main result is the proof of the existence of a solution of the Beltrami differential equation in $\mathbb{C}$

$$
\frac{\partial}{\partial \bar{z}} w(\tau ; z, \bar{z})=\tau \mu(z, \bar{z}) \frac{\partial}{\partial z} w(\tau ; z, \bar{z})
$$

where $\mu(z, \bar{z})$ is a Beltrami differential with $\|\mu(z, \bar{z})\|_{\infty} \leq c<1$. The solution of the Beltrami differential equation is based on an iteration method due to Bojarski [1955], while it was Morrey [1938] who first proved the existence of the solution of the Beltrami equation. One of the main ingredients in the proof of the convergence of the Bojarski iteration method is the $L^{2}$-isometry of the inverse $\bar{\partial}^{-1}$ of the $\bar{\partial}$ operator in one complex variable. Kuranishi generalized the iteration method of Bojarski and constructed the Kuranishi map $\kappa: \mathbb{H}^{0,1}\left(X, T_{X}^{1,0}\right) \rightarrow \mathbb{H}^{0,2}\left(X, T_{X}^{1,0}\right)$, the most basic technical tool in various aspects of deformation theory.

Fix a basis $\left\{\mu_{i}\right\}_{i=1}^{n}$ of harmonic $T_{X_{p}}^{(1,0)}$-valued $(0,1)$ forms with

$$
\sum_{i=1}^{n}\left\|\mu_{i}\right\|_{L^{\infty}} \leq 1
$$

and let $\mu(t)=\sum_{i=1}^{n} t_{i} \mu_{i}$ be the integrable Beltrami differential of the Kuranishi family $\varpi: \mathscr{X} \rightarrow \Delta_{p, 1}$.

Theorem 3.2. The $(1,0)$ form $\eta(t)$ on $X_{p}$ constructed in Theorem 2.1 converges in $L^{2}$-norm as long as $|t|<1$.

Proof. Recall that

is constructed as

$$
\eta(t)=\theta+\sum_{i=1}^{n} t_{i} \eta_{i}+\sum_{|I| \geq 2} t^{I} \eta_{I}
$$

$$
\left\{\begin{array}{l}
\left.\eta_{i}=-\mathbb{G} \bar{\partial}^{*} \partial\left(\mu_{i}\right\lrcorner \theta\right), \\
\left.\eta_{\left(i_{1}, \ldots, i_{n}\right)}=-\mathbb{G} \bar{\partial}^{*} \partial\left(\sum_{k=1}^{n} \mu_{k}\right\lrcorner \eta_{\left(i_{1}, \ldots, i_{k}-1, \ldots, i_{n}\right)}\right) .
\end{array}\right.
$$

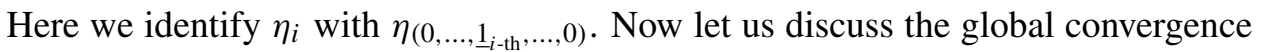
in $L^{2}$-norm of the power series. By the quasi-isometry result in Proposition 3.1, together with (3-2) and the assumption $\sum_{i=1}^{n}\left\|\mu_{i}\right\|_{L^{\infty}} \leq 1$, we have

$$
\sum_{|I|=i}\left\|\eta_{I}\right\|_{L^{2}} \leq\left(\sum_{i=1}^{n}\left\|\mu_{i}\right\|_{L^{\infty}}\right)\left(\sum_{|I|=i-1}\left\|\eta_{I}\right\|_{L^{2}}\right) .
$$

Also when $|I|=1$, it is clear that

$$
\sum_{i=1}^{n}\left\|\eta_{i}\right\|_{L^{2}} \leq\left(\sum_{i=1}^{n}\left\|\mu_{i}\right\|_{L^{\infty}}\right)\|\theta\|_{L^{2}} \leq\|\theta\|_{L^{2}} .
$$


By induction, this yields that for every $k \geq 1$,

$$
\sum_{|I|=k}\left\|\eta_{I}\right\|_{L^{2}} \leq\|\theta\|_{L^{2}}
$$

which implies the estimates of $\eta(t)$

$$
\|\eta(t)\|_{L^{2}} \leq\|\theta\|_{L^{2}}+\|\theta\|_{L^{2}} \sum_{\|I\| \geq 1}|t|^{|I|} .
$$

Corollary 3.3. The function $\theta(t)$ constructed in Theorem 2.1 converges in $L^{2}$-norm for $|t|<1$.

Proof. This follows directly from Theorem 3.2.

\section{The Siegel metric and its curvature}

Let us recall the local and global Torelli theorems of the moduli spaces of compact Riemann surfaces with genus $g$. Denote the Teichmüller space of the compact Riemann surface of genus $g$ by $\mathscr{T}_{g}$ and the generalized Siegel upper half-plane by $\mathscr{H}_{g}$, which is $\left\{Z \in \mathrm{M}(g, \mathbb{C}) \mid Z=Z^{t}, \operatorname{Im} Z>0\right\}$, an irreducible noncompact symmetric space, giving $\mathscr{A}_{g}$ a locally symmetric structure. Then

$$
\Gamma_{g}(n):=\operatorname{Kernel}\left(\Gamma_{g} \stackrel{\rho}{\rightarrow} \operatorname{Sp}(g, \mathbb{Z}) \stackrel{\pi}{\rightarrow} \operatorname{Sp}(g, \mathbb{Z} / n \mathbb{Z})\right)
$$

for $n \geq 2$ and $T_{g}:=\operatorname{Kernel}\left(\Gamma_{g} \stackrel{\rho}{\rightarrow} \operatorname{Sp}(g, \mathbb{Z})\right)$, where $\Gamma_{g}$ is the mapping class group of the compact Riemann surface of genus $g$. Also, the moduli space $\mu_{g}^{(n)}$ of the compact Riemann surface of genus $g$ with a fixed $n$-level structure is defined as the quotient of $\mathscr{T}_{g}$ by $\Gamma_{g}(n)$. We will fix $n \geq 3$ from now on. Meanwhile, the Torelli space $\mathcal{T}_{o} r_{g}$ is the quotient of $\mathcal{T}_{g}$ by $T_{g}$, called the Torelli group. Then we have the commutative diagram

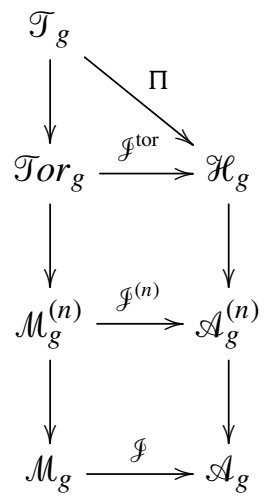

$\mathscr{F}$ is always injective for $g \geq 2$. $\mathscr{g}^{\text {tor }}$ is an open embedding for $g=2$, while $g^{\text {tor }}$ and $\mathscr{g}^{(n)}$ are $2: 1$ branched coverings onto its image ramified over hyperelliptic locus 
for $g \geq 3$. In other words, $g^{\text {tor }}: \mathscr{T}_{g} / \widetilde{T}_{g} \rightarrow \mathscr{H}_{g}$ is an embedding where $\widetilde{T}_{g}$ is defined as $\rho^{-1}\left(\left\langle-I_{2 g}\right\rangle\right)$ and $\left\langle-I_{2 g}\right\rangle$ is a subgroup of $\operatorname{Sp}(g, \mathbb{Z})$ generated by $-I_{2 g}$. We shift to the local point of view. $\Pi$ is everywhere an immersion for $g=2$, but for the case $g \geq 3$, the tangent map of $\Pi$ is injective on the nonhyperelliptic locus and vanishes on the normal directions of the hyperelliptic locus $\mathscr{H}_{\mathscr{E}} \mathscr{\mathscr { T }} \mathcal{T}_{g}$. When restricted to $\mathscr{H}_{\mathscr{E}} \mathscr{T}_{g}, \Pi$ is an immersion. According to [Liu et al. 2012b], the tangent map of $\mathscr{g}^{\text {tor }}: \mathscr{T}_{g} / \widetilde{T}_{g} \rightarrow \mathscr{H}_{g}$ at the hyperelliptic locus from the Zariski tangent space of $\mathscr{T}_{g} / \widetilde{T}_{g}$ to the tangent space of $\mathscr{H}_{g}$ is injective.

Denote the Hodge bundle on $M_{g}$ and $\mathscr{A}_{g}$ by $\mathscr{E}_{g}$; its fiber at a point is the vector space of holomorphic one-forms on $[X]$, a representative of the complex structure given by that point. There are three canonical metrics on $\mathscr{H}_{g}$ and $\mathscr{A}_{g}$, namely the Hodge metric, the Bergman metric and the Siegel metric. Hard Lefschetz decomposition and Hodge polarization give us a hermitian metric on $\mathscr{E}_{g}$, denoted by $\langle$,$\rangle . From the natural isomorphism \Omega_{\mathscr{H}_{g}}^{1} \cong S^{2 \mathscr{E}_{g}}$, where $S$ is the symmetric operator, it induces a hermitian metric on $T_{\mathscr{H}_{g}}^{(1,0)}$, denoted by $\tilde{\omega}_{h}$. The Bergman metric is defined by the Bergman kernel

$$
\rho=-\log \operatorname{det}\left(\mathbb{1}_{g}-\overline{W^{t}} W\right)=-\log \operatorname{det}\left(\mathbb{1}_{g}-\overline{\left(\frac{\mathbb{1}_{g}+i Z}{\mathbb{1}_{g}-i Z}\right)^{t}}\left(\frac{\mathbb{1}_{g}+i Z}{\mathbb{1}_{g}-i Z}\right)\right),
$$

where $W \in\left\{A \mid A \in \mathrm{M}(g, \mathbb{C}), A^{t}=A, \mathbb{1}_{g}-\overline{A^{t}} A>0\right\}$, which is the bounded domain, and $Z$ is the coordinate of the Siegel upper half-plane

$$
\left\{Z \mid Z \in \mathrm{M}(g, \mathbb{C}), Z=Z^{t}, \operatorname{Im}(Z)>0\right\} .
$$

Here $\mathbf{M}(g, \mathbb{C})$ denotes the group of complex $g \times g$ matrices. Thus $\tilde{\omega}_{b}=\frac{i}{2} \partial \bar{\partial} \rho$. Finally, the Siegel metric $\tilde{\omega}_{s}$ is defined by $\pi c_{1}\left(\mathscr{E}_{g},\langle\rangle,\right)$. Pulled back by the period map, Siegel metrics, denoted by $\omega_{s}$, also exist on $\mathscr{T}_{g}, \mathscr{T}_{g}$ and $\mathcal{M}_{g}$.

These three metrics are Kähler metrics and also invariant metrics on the irreducible homogeneous and symmetric space $\mathscr{H}_{g}$. It is clear that they are different by a constant multiple, while by [Yin 2010, Theorem 3.1 of Chapter 4], we know they are actually the same on $\mathscr{H}_{g}$.

Definition 4.1 (symmetric derivatives $S_{i}, S_{\bar{j}}, S_{i \bar{j}}, S_{i \bar{j}} S_{k \bar{l}}$ and $S_{i \bar{j}}^{\prime} S_{k \bar{l}}^{\prime}$ ). We give some examples to explain the use of these symbols. Here we use the notation $A:=A(t)$, and similarly for $B(t), C(t)$ and $D(t)$.

(1) First derivative: $S_{i}, S_{\bar{j}}$ and $S_{i \bar{j}}$.

$S_{i}(A \bar{B} C \bar{D}):=\frac{\partial A}{\partial t_{i}} \bar{B} C \bar{D}+A \bar{B} \frac{\partial C}{\partial t_{i}} \bar{D}, \quad S_{\bar{j}}(A \bar{B} C \bar{D}):=A \frac{\overline{\partial B}}{\partial t_{j}} C \bar{D}+A \bar{B} C \frac{\overline{\partial D}}{\partial t_{j}}$, $S_{i \bar{j}}(A \bar{B} C \bar{D}):=\frac{\partial A}{\partial t_{i}} \frac{\overline{\partial B}}{\partial t_{j}} C \bar{D}+\frac{\partial A}{\partial t_{i}} \bar{B} C \frac{\overline{\partial D}}{\partial t_{j}}+A \frac{\overline{\partial B}}{\partial t_{j}} \frac{\partial C}{\partial t_{i}} \bar{D}+A \bar{B} \frac{\partial C}{\partial t_{i}} \frac{\overline{\partial D}}{\partial t_{j}}$, 
where $A, B, C, D \in \mathrm{M}(n, \mathbb{C})$ are all holomorphic in $t$. Indices without a bar mean taking derivatives through all holomorphic matrices, and indices with a bar do so through all antiholomorphic matrices.

(2) Second derivative: $\boldsymbol{S}_{i \bar{j}} \boldsymbol{S}_{k \bar{l}}$ and $\boldsymbol{S}_{i \bar{j}}^{\prime} \boldsymbol{S}_{k \bar{l}}^{\prime}$.

$S_{i j} S_{k \bar{l}}(A \bar{B} C \bar{D})$

$$
\begin{aligned}
& :=\frac{\partial^{2} A}{\partial t_{i} \partial t_{k}} \overline{\frac{\partial^{2} B}{\partial t_{j} \partial t_{l}}} C \bar{D}+\frac{\partial A}{\partial t_{i}} \overline{\frac{\partial^{2} B}{\partial t_{j} \partial t_{l}}} \frac{\partial C}{\partial t_{k}} \bar{D}+\frac{\partial A}{\partial t_{k}} \overline{\frac{\partial^{2} B}{\partial t_{j} \partial t_{l}}} \frac{\partial C}{\partial t_{i}} \bar{D}+\overline{A \frac{\partial^{2} B}{\partial t_{j} \partial t_{l}}} \frac{\partial^{2} C}{\partial t_{i} \partial t_{k}} \bar{D} \\
& +\frac{\partial^{2} A}{\partial t_{i} \partial t_{k}} \frac{\overline{\partial B}}{\partial t_{j}} C \frac{\overline{\partial D}}{\partial t_{l}}+\frac{\partial A}{\partial t_{i}} \frac{\overline{\partial B}}{\partial t_{j}} \frac{\partial C}{\partial t_{k}} \frac{\overline{\partial D}}{\partial t_{l}}+\frac{\partial A}{\partial t_{k}} \frac{\overline{\partial B}}{\partial t_{j}} \frac{\partial C}{\partial t_{i}} \frac{\overline{\partial D}}{\partial t_{l}}+A \frac{\overline{\partial B}}{\partial t_{j}} \frac{\partial^{2} C}{\partial t_{i} \partial t_{k}} \frac{\overline{\partial D}}{\partial t_{l}} \\
& +\frac{\partial^{2} A}{\partial t_{i} \partial t_{k}} \frac{\overline{\partial B}}{\partial t_{l}} C \frac{\overline{\partial D}}{\partial t_{j}}+\frac{\partial A}{\partial t_{i}} \frac{\overline{\partial B}}{\partial t_{l}} \frac{\partial C}{\partial t_{k}} \frac{\overline{\partial D}}{\partial t_{j}}+\frac{\partial A}{\partial t_{k}} \frac{\overline{\partial B}}{\partial t_{l}} \frac{\partial C}{\partial t_{i}} \frac{\overline{\partial D}}{\partial t_{j}}+A \frac{\overline{\partial B}}{\partial t_{l}} \frac{\partial^{2} C}{\partial t_{i} \partial t_{k}} \frac{\overline{\partial D}}{\partial t_{j}} \\
& +\frac{\partial^{2} A}{\partial t_{i} \partial t_{k}} \bar{B} C \overline{\frac{\partial^{2} D}{\partial t_{j} \partial t_{l}}}+\frac{\partial A}{\partial t_{i}} \bar{B} \frac{\partial C}{\partial t_{k}} \overline{\frac{\partial^{2} D}{\partial t_{j} \partial t_{l}}}+\frac{\partial A}{\partial t_{k}} \bar{B} \frac{\partial C}{\partial t_{i}} \overline{\frac{\partial^{2} D}{\partial t_{j} \partial t_{l}}}+A \bar{B} \frac{\partial^{2} C}{\partial t_{i} \partial t_{k}} \overline{\frac{\partial^{2} D}{\partial t_{j} \partial t_{l}}},
\end{aligned}
$$

$S_{i j}^{\prime} S_{k \bar{l}}^{\prime}(A \bar{B} C \bar{D})$

$$
:=\frac{\partial A}{\partial t_{i}} \frac{\overline{\partial B}}{\partial t_{j}} \frac{\partial C}{\partial t_{k}} \frac{\overline{\partial D}}{\partial t_{l}}+\frac{\partial A}{\partial t_{k}} \frac{\overline{\partial B}}{\partial t_{j}} \frac{\partial C}{\partial t_{i}} \frac{\overline{\partial D}}{\partial t_{l}}+\frac{\partial A}{\partial t_{i}} \frac{\overline{\partial B}}{\partial t_{l}} \frac{\partial C}{\partial t_{k}} \frac{\overline{\partial D}}{\partial t_{j}}+\frac{\partial A}{\partial t_{i}} \frac{\overline{\partial B}}{\partial t_{l}} \frac{\partial C}{\partial t_{k}} \frac{\overline{\partial D}}{\partial t_{j}} .
$$

The difference between these two symbols lies in that $\frac{\partial}{\partial t_{i}}$ and $\frac{\partial}{\partial t_{k}}$ can't operate on a matrix simultaneously in $S_{i j}^{\prime} S_{k \bar{l}}^{\prime}$.

Theorem 4.2. The Siegel metric $\omega_{s}(t)$ on the nonhyperelliptic locus of $\mathscr{T}_{g}$ can be written as

$$
\omega_{s}(t)=\frac{\mathrm{i}}{2} \sum_{n=1}^{\infty} \frac{1}{n} \partial \bar{\partial} \operatorname{tr}(A(t) \overline{A(t)})^{n} .
$$

Proof. From the definition of the Siegel metric and the fact that holomorphic one-forms on the Riemann surface and its Jacobian torus can be identified, we will write it out explicitly using the Kuranishi coordinate $\Delta_{p, \epsilon}$ on $\mathscr{T}_{g}$ with $g \geq 3$ and the deformation formula for holomorphic one-forms, where $p$ lies in the nonhyperelliptic locus.

$$
\begin{aligned}
\omega_{s}(t) & =\pi c_{1}\left(\mathscr{E}_{g},\langle\rangle\right) \\
& =-\frac{\mathrm{i}}{2} \partial \bar{\partial} \log \operatorname{det} \frac{\mathrm{i}}{2} \int_{X_{p}} \theta^{\alpha}(t) \wedge \overline{\theta^{\beta}(t)} \\
& =-\frac{\mathrm{i}}{2} \partial \bar{\partial} \operatorname{tr} \log \frac{\mathrm{i}}{2} \int_{X_{p}}\left(\theta_{p}^{\alpha}+A(t)_{\gamma}^{\alpha} \bar{\theta}_{p}^{\gamma}+E^{\alpha}(t)\right) \wedge \overline{\left(\theta_{p}^{\beta}+A(t)_{\delta}^{\beta} \bar{\theta}_{p}^{\delta}+E^{\beta}(t)\right)} \\
& =-\frac{\mathrm{i}}{2} \partial \bar{\partial} \operatorname{tr} \log \left(M_{p, \alpha \beta}-A(t)_{\gamma}^{\alpha} \overline{A(t)_{\delta}^{\beta}} M_{p, \delta \gamma}\right)
\end{aligned}
$$




$$
\begin{aligned}
& =-\frac{\mathrm{i}}{2} \partial \bar{\partial} \operatorname{tr} \log M_{p, \alpha \eta}\left(\delta_{\eta \beta}-M_{p}^{\eta \sigma} A(t)_{\gamma}^{\sigma} \overline{A(t)_{\delta}^{\beta}} M_{p, \delta \gamma}\right) \\
& =-\frac{\mathrm{i}}{2} \partial \bar{\partial} \operatorname{tr} \log \left(\delta_{\alpha \beta}-M_{p}^{\alpha \sigma} A(t)_{\gamma}^{\sigma} \overline{A(t)_{\delta}^{\beta}} M_{p, \delta \gamma}\right) .
\end{aligned}
$$

We remark here that the $\frac{\mathrm{i}}{2} \int_{X_{p}} \theta^{\alpha}(t) \wedge \overline{\theta^{\beta}(t)}$ are positive hermitian matrices for $t$ small, and thus diagonalizable matrices. Thus it makes sense for the operator tr log.

Formulate all these into the matrix type to get

$$
\omega_{s}(t)=-\frac{\mathrm{i}}{2} \partial \bar{\partial} \operatorname{tr} \log \left(I-M_{p}^{-1} A(t) M_{p} \overline{A(t)}^{T}\right) .
$$

From the Hodge-Riemann bilinear relation $A(t) M_{p}=M_{p} A(t)^{T}$, it follows that

$$
M_{p}^{-1} A(t) M_{p} \overline{A(t)}^{T}=A(t)^{T} \overline{A(t)}^{T} .
$$

Then the Siegel metric $\omega_{s}(t)$ is given by

$$
\begin{aligned}
\omega_{s}(t) & =-\frac{\mathrm{i}}{2} \partial \bar{\partial} \operatorname{tr} \log \left(I-A(t)^{T} \overline{A(t)}^{T}\right)=\frac{\mathrm{i}}{2} \partial \bar{\partial} \operatorname{tr} \sum_{n=1}^{\infty} \frac{1}{n}\left(A(t)^{T} \overline{A(t)}^{T}\right)^{n} \\
& =\frac{\mathrm{i}}{2} \sum_{n=1}^{\infty} \frac{1}{n} \partial \bar{\partial} \operatorname{tr}\left(A(t)^{T} \overline{A(t)}^{T}\right)^{n}=\frac{\mathrm{i}}{2} \sum_{n=1}^{\infty} \frac{1}{n} \partial \bar{\partial} \operatorname{tr}(A(t) \overline{A(t)})^{n} .
\end{aligned}
$$

Restricted to the origin, the Siegel metric is $\omega_{s}(0)=\frac{\mathrm{i}}{2} \sum_{i, j=1}^{n} \operatorname{tr}\left(A_{i} \overline{A_{j}}\right) d t_{i} \wedge d \bar{t}_{j}$.

To compute the curvature of the Siegel metric, we rewrite (3-2) according to the degree of $t$ :

$$
\begin{aligned}
\omega_{s}(t) & =\frac{\mathrm{i}}{2} \partial \bar{\partial} \sum_{k \geq 2} \sum_{\substack{m_{i}>0,1 \leq i \leq 2 l \\
m_{1}+\cdots+m_{2 l}=k}} \frac{1}{l} \operatorname{tr}\left(A_{m_{1}}(t) \overline{A_{m_{2}}(t)} \ldots \overline{A_{m_{2 l}}(t)}\right) \\
& =\frac{\mathrm{i}}{2} \sum_{k \geq 2} \sum_{\substack{m_{i}>0,1 \leq i \leq 2 l \\
m_{1}+\cdots+m_{2 l}=k}} \frac{1}{l} \operatorname{tr}\left(S_{i \bar{j}}\left(A_{m_{1}}(t) \overline{A_{m_{2}}(t)} \ldots \overline{A_{m_{2 l}}(t)}\right)\right) d t_{i} \wedge d \bar{t}_{j} \\
& =\frac{\mathrm{i}}{2} \sum_{k \geq 0} \sum_{\substack{m_{i}>0,1 \leq i \leq 2 l \\
m_{1}+\cdots+m_{2 l}=k+2}} \frac{1}{l} \operatorname{tr}\left(S_{i \bar{j}}\left(A_{m_{1}}(t) \overline{A_{m_{2}}(t)} \ldots \overline{A_{m_{2 l}}(t)}\right)\right) d t_{i} \wedge d \bar{t}_{j}
\end{aligned}
$$

From (4-3), we know that, if we set $\omega_{s}(t)=\frac{\mathrm{i}}{2} \omega_{i \bar{j}} d t_{i} \wedge d \bar{t}_{j}$, then

$$
\omega_{i \bar{j}}=\sum_{k \geq 0} \sum_{\substack{m_{i}>0,1 \leq i \leq 2 l \\ m_{1}+\cdots+m_{2 l}=k+2}} \frac{1}{l} \operatorname{tr}\left(S_{i \bar{j}}\left(A_{m_{1}}(t) \overline{A_{m_{2}}(t)} \ldots \overline{A_{m_{2 l}}(t)}\right)\right),
$$

with $\omega_{i \bar{j}}(0)=\operatorname{tr}\left(A_{i} \overline{A_{j}}\right)$. 
We need an auxiliary combinatorial lemma before getting to the curvature formula.

Lemma 4.3. The function $h_{i j}(t)$ has the expansion

$$
\delta_{i j}+\left(h_{i j}\right)_{1}+\left(h_{i j}\right)_{2}+\cdots,
$$

where $\left(h_{i j}\right)_{n}$ is the $n$-th order part of the expansion; then $h^{i j}(t)$ can be expanded as

$$
h^{i j}(t)=\delta_{i j}-\left(h_{i j}\right)_{1}+\sum_{k \geq 2} \sum_{\substack{m_{i}>0,1 \leq i \leq l \\ m_{1}+\cdots+m_{l}=k}}(-1)^{l}\left(h_{i i_{1}}\right)_{m_{1}}\left(h_{i_{1} i_{2}}\right)_{m_{2}} \ldots\left(h_{i_{l-1} j}\right)_{m_{l}} .
$$

Proof. Directly check that $h_{i j}(t) h^{j k}(t)=\delta_{i k}$, which is equivalent to

$$
\begin{aligned}
\delta_{i k}=\left[\delta_{i j}\right. & \left.+\left(h_{i j}\right)_{1}+\sum_{p \geq 2}\left(h_{i j}\right)_{p}\right] \\
& \times\left[\delta_{j k}-\left(h_{j k}\right)_{1}+\sum_{p \geq 2} \sum_{\substack{m_{i}>0,1 \leq i \leq l \\
m_{1}+\cdots+m_{l}=p}}(-1)^{l}\left(h_{j i_{1}}\right)_{m_{1}}\left(h_{i_{1} i_{2}}\right)_{m_{2}} \ldots\left(h_{i_{l-1} k}\right)_{m_{l}}\right] .
\end{aligned}
$$

It is quite easy to see that the zeroth- and first-order parts of both sides coincide. Thus this reduces to checking that for $p \geq 2$,

$0=\left(h_{i k}\right)_{p}-\left(h_{i j}\right)_{p-1}\left(h_{j k}\right)_{1}+\cdots$

$$
\begin{aligned}
& +\left(h_{i j}\right)_{1} \sum_{\substack{m_{i}>0,1 \leq i \leq l \\
m_{1}+\cdots+m_{l}=p-1}}(-1)^{l}\left(h_{j i_{1}}\right)_{m_{1}}\left(h_{i_{1} i_{2}}\right)_{m_{2}} \ldots\left(h_{i_{l-1} k}\right)_{m_{l}} \\
& +\sum_{\substack{m_{i}>0,1 \leq i \leq l \\
m_{1}+\cdots+m_{l}=p}}(-1)^{l}\left(h_{i i_{1}}\right)_{m_{1}}\left(h_{i_{1} i_{2}}\right)_{m_{2}} \ldots\left(h_{i_{l-1} k}\right)_{m_{l}} .
\end{aligned}
$$

The right-hand side can be written as

$$
\begin{gathered}
\sum_{\substack{i=1 \\
m_{1}=i, m_{j}>0,2 \leq j \leq l \\
m_{1}+\cdots+m_{l}=p}}^{p}(-1)^{l-1}\left(h_{i i_{1}}\right)_{m_{1}}\left(h_{i_{1} i_{2}}\right)_{m_{2}} \ldots\left(h_{i_{l-1} k}\right)_{m_{l}} \\
+\sum_{\substack{m_{i}>0,1 \leq i \leq l \\
m_{1}+\cdots+m_{l}=p}}(-1)^{l}\left(h_{i i_{1}}\right)_{m_{1}}\left(h_{i_{1} i_{2}}\right)_{m_{2}} \ldots\left(h_{i_{l-1} k}\right)_{m_{l}} \\
=\sum_{\substack{m_{i}>0,1 \leq i \leq l \\
m_{1}+\cdots+m_{l}=p}}(-1)^{l-1}\left(h_{i i_{1}}\right)_{m_{1}}\left(h_{i_{1} i_{2}}\right)_{m_{2}} \ldots\left(h_{i_{l-1} k}\right)_{m_{l}} \\
+\sum_{\substack{m_{i}>0,1 \leq i \leq l \\
m_{1}+\cdots+m_{l}=p}}(-1)^{l}\left(h_{i i_{1}}\right)_{m_{1}}\left(h_{i_{1} i_{2}}\right)_{m_{2}} \ldots\left(h_{i_{l-1} k}\right)_{m_{l}} .
\end{gathered}
$$

Now clearly this is zero. Our lemma is proved. 
Choose a normal coordinate around $p$ such that $\omega_{i \bar{j}}(0)=\delta_{i j},\left(\partial \omega_{i j} / \partial t_{k}\right)(0)=$ $\left(\partial \omega_{i \bar{j}} / \partial \bar{t}_{k}\right)(0)=0$, and $\left(\partial \omega_{i \bar{j}} / \partial t_{k} \partial t_{l}\right)(0)=\left(\partial \omega_{i \bar{j}} / \partial \bar{t}_{k} \partial \bar{t}_{l}\right)(0)=0$, still denoted by $\Delta_{p, \epsilon}$. According to the convention of $A_{N}(t)$ we make after the definition of $A(t)$ and $E(t)$, this is equivalent to saying

$$
\left\{\begin{array}{l}
\operatorname{tr}\left(A_{i} \overline{A_{j}}\right)=\delta_{i j}, \\
\operatorname{tr}\left(A_{i} \overline{A_{j k}}\right)=\operatorname{tr}\left(A_{i k} \overline{A_{j}}\right)=0, \\
\operatorname{tr}\left(A_{i k l} \overline{A_{j}}\right)=\operatorname{tr}\left(A_{i} \overline{A_{j k l}}\right)=0 .
\end{array}\right.
$$

From Lemma 4.3, we get

$$
\begin{aligned}
& \omega^{\bar{i} j}=\delta_{i j}+\sum_{k \geq 1} \sum_{\substack{m_{i}>0,1 \leq i \leq l \\
m_{1}+\cdots+m_{l}=k}}(-1)^{l} \prod_{i=1}^{l} \frac{1}{s_{i}} \\
& \times\left(\sum_{\substack{m_{1 n}>0,1 \leq n \leq 2 s_{1} \\
\sum_{n=1}^{2 s_{1}} m_{1 n}=m_{1}+2}} \operatorname{tr}\left(S_{i \overline{i_{1}}}\left(A_{m_{11}}(t) \ldots \overline{\left.A_{m_{12 s_{1}}}(t)\right)}\right)\right)\right. \\
& \times\left(\sum_{\substack{m_{2 n}>0,1 \leq n \leq 2 s_{2} \\
\sum_{n=1}^{2 s_{2}} m_{2 n}=m_{2}+2}} \operatorname{tr}\left(\boldsymbol{S}_{i_{1} \overline{\bar{i}_{2}}}\left(A_{m_{21}}(t) \ldots \overline{A_{m_{22 s_{2}}}(t)}\right)\right)\right) \\
& \times \times\left(\sum_{\substack{m_{l n}>0,1 \leq n \leq 2 s_{l} \\
\sum_{n=1}^{2 s_{l}} m_{l n}=m_{l}+2}} \operatorname{tr}\left(S_{i_{l-1} \bar{j}}\left(A_{m_{l 1}}(t) \ldots \overline{A_{m_{l 2 s_{l}}}(t)}\right)\right)\right) .
\end{aligned}
$$

Theorem 4.4. The curvature $\mathrm{R}_{i \bar{j} k \bar{l}}$ of the Siegel metric $\omega_{s}(t)$ is given by

$$
\begin{aligned}
& \mathrm{R}_{i \bar{j} k \bar{l}}=-\sum_{N \geq 0} \sum_{\substack{m_{i}>0,1 \leq i \leq 2 l \\
m_{1}+\cdots+m_{2 l}=N+4}} \frac{1}{l} \operatorname{tr}\left(\boldsymbol{S}_{i \bar{j}} \boldsymbol{S}_{k \bar{l}}\left(A_{m_{1}}(t) \overline{A_{m_{2}}(t)} \ldots \overline{A_{m_{2 l}}(t)}\right)\right) \\
& +\sum_{N \geq 0} \sum_{\substack{N_{i} \geq 0,1 \leq i \leq 3 \\
\sum_{i=1}^{3} N_{i}=N}}\left[\sum_{\substack{m_{i}>0,1 \leq i \leq l \\
m_{1}+\cdots+m_{l}=N_{1}}}(-1)^{l} \prod_{i=1}^{l} \frac{1}{s_{i}}\right. \\
& \times \sum_{m_{1 n}>0,1 \leq n \leq 2 s_{1}} \operatorname{tr}\left(S_{q \overline{i_{1}}}\left(A_{m_{11}}(t) \cdots \overline{A_{m_{12 s_{1}}}(t)}\right)\right) \\
& \sum_{n=1}^{2 s_{1}} m_{1 n}=m_{1}+2 \\
& \times \sum_{m_{2 n}>0,} \operatorname{tr}\left(S_{i_{1} \overline{i_{2}}}\left(A_{m_{21}}(t) \ldots \overline{A_{m_{22 s_{2}}}(t)}\right)\right) \ldots \\
& \sum_{n=1}^{2 s_{2}} m_{2 n}=m_{2}+2 \\
& \left.\times \sum_{m_{l n}>0,} \operatorname{tr}\left(S_{i_{l-1} \bar{p}}\left(A_{m_{l 1}}(t) \ldots \overline{A_{m_{l s_{l}}}(t)}\right)\right)\right] \\
& \sum_{n=1}^{2 s_{l}} m_{l n}=m_{l}+2
\end{aligned}
$$




$$
\begin{aligned}
& \times\left[\sum_{\substack{m_{i}>0, \sum_{i=1}^{2 l} m_{i}=N_{2}+3}} \frac{1}{l} \operatorname{tr}\left(S_{i} S_{k \bar{q}}\left(A_{m_{1}}(t) \ldots \overline{A_{m_{2 l}}(t)}\right)\right)\right] \\
& \times\left[\sum_{\substack{m_{i}>0, \sum_{i=1}^{2 l} m_{i}=N_{3}+3}} \frac{1}{l} \operatorname{tr}\left(S_{\bar{j}} S_{p \bar{l}}\left(A_{m_{1}}(t) \ldots \overline{A_{m_{2 l}}(t)}\right)\right)\right],
\end{aligned}
$$

where we need the convention that the first square bracket in the second summand will be $\delta_{q p}$ as $N_{1}=0$.

Proof. Just use the well known curvature formula

$$
\mathrm{R}_{i \bar{j} k \bar{l}}=-\frac{\partial^{2} \omega_{k \bar{l}}}{\partial t_{i} \partial \bar{t}_{j}}+\omega^{\bar{q} p} \frac{\partial \omega_{k \bar{q}}}{\partial t_{i}} \frac{\partial \omega_{p \bar{l}}}{\partial \bar{t}_{j}}
$$

By use of (4-4), we have

$$
\begin{aligned}
\frac{\partial^{2} \omega_{k \bar{l}}}{\partial t_{i} \partial \bar{t}_{j}} & =\sum_{n \geq 0} \sum_{\substack{m_{i}>0,1 \leq i \leq 2 l \\
m_{1}+\cdots+m_{2 l}=n+4}} \frac{1}{l} \operatorname{tr}\left(S_{i j} S_{k \bar{l}}\left(A_{m_{1}}(t) \overline{A_{m_{2}}(t)} \ldots \overline{A_{m_{2 l}}(t)}\right)\right), \\
\frac{\partial \omega_{k \bar{q}}}{\partial t_{i}} & =\sum_{n \geq 0} \sum_{\substack{m_{i}>0,1 \leq i \leq 2 l \\
m_{1}+\cdots+m_{2 l}=n+3}} \frac{1}{l} \operatorname{tr}\left(\boldsymbol{S}_{i} \boldsymbol{S}_{k \bar{q}}\left(A_{m_{1}}(t) \overline{A_{m_{2}}(t)} \ldots \overline{A_{m_{2 l}}(t)}\right)\right), \\
\frac{\partial \omega_{p \bar{l}}}{\partial \bar{t}_{j}} & =\sum_{n \geq 0} \sum_{\substack{m_{i}>0,1 \leq i \leq 2 l \\
m_{1}+\cdots+m_{2 l}=n+3}} \frac{1}{l} \operatorname{tr}\left(\boldsymbol{S}_{\bar{j}} \boldsymbol{S}_{p \bar{l}}\left(A_{m_{1}}(t) \overline{A_{m_{2}}(t)} \ldots \overline{A_{m_{2 l}}(t)}\right)\right) .
\end{aligned}
$$

From this we get the formula (4-6) of $\omega^{\bar{i} j}$. Thus the theorem follows easily.

Also the curvature of the Siegel metric at the point $p$ can be easily gotten from the curvature formula together with (4-5):

$$
\begin{aligned}
\mathrm{R}_{i \bar{j} k \bar{l}}(0)= & -\operatorname{tr}\left(S_{i \bar{j}} S_{k \bar{l}}\left(A_{2}(t) \overline{A_{2}(t)}\right)\right)-\frac{1}{2} \operatorname{tr}\left(S_{i j} S_{k \bar{l}}\left(A_{1}(t) \overline{A_{1}(t)} A_{1}(t) \overline{A_{1}(t)}\right)\right) \\
& +\operatorname{tr}\left(S_{i} S_{k \bar{p}}\left(A_{2}(t) \overline{A_{1}(t)}\right)\right) \operatorname{tr}\left(S_{\bar{j}} S_{p \bar{l}}\left(A_{1}(t) \overline{A_{2}(t)}\right)\right) \\
= & -\operatorname{tr}\left(A_{i k} \overline{A_{j l}}\right)-\operatorname{tr}\left(A_{i} \overline{A_{j}} A_{k} \overline{A_{l}}\right) \\
& -\operatorname{tr}\left(A_{i} \overline{A_{l}} A_{k} \overline{A_{j}}\right)+\operatorname{tr}\left(A_{i k} \overline{A_{p}}\right) \operatorname{tr}\left(A_{p} \overline{A_{j l}}\right) \\
= & -\operatorname{tr}\left(A_{i k} \overline{A_{j l}}\right)-\operatorname{tr}\left(A_{i} \overline{A_{j}} A_{k} \overline{A_{l}}\right)-\operatorname{tr}\left(A_{i} \overline{A_{l}} A_{k} \overline{A_{j}}\right) .
\end{aligned}
$$
The holomorphic sectional curvature along the direction $v=\sum_{i=1}^{3 g-3} a_{i} \mu_{i} \in \mathbb{H}_{\bar{\partial}}^{0,1}\left(X_{p}, T_{X_{p}}\right)$
is given by 


$$
\begin{aligned}
\mathrm{H}(v) & =\frac{\mathrm{R}_{v \bar{v} v \bar{v}}}{|v|^{4}}=\frac{\sum_{i, j, k, l=1}^{3 g-3} a_{i} \overline{a_{j}} a_{k} \overline{a_{l}} \mathrm{R}_{i \bar{j} k \bar{l}}(0)}{\left(\sum_{i, j=1}^{3 g-3} a_{i} \overline{a_{j}} \omega_{i \bar{j}}(0)\right)^{2}} \\
& =\frac{-2 \operatorname{tr}\left(\left(a_{i} A_{i}\right) \overline{\left(a_{j} A_{j}\right)}\left(a_{k} A_{k}\right) \overline{\left(a_{l} A_{l}\right)}\right)-\operatorname{tr}\left(\left(a_{i} a_{k} A_{i k}\right)\left(\overline{a_{j} a_{l} A_{j l}}\right)\right)}{\left(a_{i} \overline{a_{j}} \operatorname{tr}\left(A_{i} \overline{A_{j}}\right)\right)^{2}} \\
& \leq-2 \frac{\operatorname{tr}\left(\left(a_{i} A_{i}\right) \overline{\left(a_{j} A_{j}\right)}\left(a_{k} A_{k}\right) \overline{\left(a_{l} A_{l}\right)}\right)}{\left(\operatorname{tr}\left(\left(a_{i} A_{i}\right) \overline{\left(a_{j} A_{j}\right)}\right)\right)^{2}} .
\end{aligned}
$$

Set $\sum_{i=1}^{3 g-3} a_{i} A_{i}=E$ and normalize $M_{p}$ to $\mathrm{i}_{g}$. Then $A(t)$ is symmetric and $A_{i}$ are all symmetric for $1 \leq i \leq g$. By the mean value inequality,

$$
1 \geq \frac{\operatorname{tr}(E \bar{E} E \bar{E})}{(\operatorname{tr}(E \bar{E}))^{2}} \geq \frac{1}{g}
$$

for the symmetric matrix $E$. The proof of Proposition 5.4 contains further details. Thus we have

$$
\mathrm{H}(v) \leq-\frac{2}{g} \text {. }
$$

\section{The second fundamental form of a nonhyperelliptic locus and the totally geodesic submanifold}

Now we are ready to compute the second fundamental form of $\mathscr{E}: \mathcal{M}_{g} \rightarrow \mathscr{A}_{g}$, always fixing the Siegel metric $\widetilde{\omega}_{s}$ on $\mathscr{A}_{g}$. Lift to $\mathscr{f}^{\text {tor }}: \mathscr{T}_{g} \rightarrow \mathscr{H}_{g}$, with Siegel metric $\widetilde{\omega}_{s}$ on $\mathscr{H}_{g}$. The local Torelli theorem assures the exact sequence

$$
0 \rightarrow T_{\mathscr{T}_{g}}^{(1,0)} \rightarrow \mathscr{J}^{\text {tor } *} T_{\mathscr{H}_{g}}^{(1,0)} \stackrel{\pi}{\rightarrow} N \rightarrow 0
$$

when restricted to a nonhyperelliptic locus of $\mathscr{T}_{g}$ and when $N$ is the normal bundle. Also we have the natural connection $\mathscr{f}^{\text {tor } *} \nabla$ on $\mathscr{f}^{\text {tor } *} T_{\mathscr{H}_{g}}^{(1,0)}$, where the Chern connection $\nabla$ is determined by $\widetilde{\omega}_{s}$ on $\mathcal{H}_{g}$. Following the argument of [Colombo and Frediani 2010, pp. 6-7], the second fundamental form $\sigma$ is defined by

$$
\sigma(s)=\pi(\nabla s), s \in A^{0}\left(T_{\mathscr{T}_{g}}^{(1,0)}\right) .
$$

From the Gauss equation, it follows that

$$
\begin{aligned}
\left(R\left(\frac{\partial}{\partial t_{k}}\right), \frac{\partial}{\partial t_{l}}\right) & \left(\frac{\partial}{\partial t_{i}}, \frac{\partial}{\partial t_{j}}\right) \\
= & \left(\widetilde{R}\left(\frac{\partial}{\partial t_{k}}\right), \frac{\partial}{\partial t_{l}}\right)\left(\frac{\partial}{\partial t_{i}}, \frac{\partial}{\partial t_{j}}\right)-\left(\sigma\left(\frac{\partial}{\partial t_{k}}\right), \sigma\left(\frac{\partial}{\partial t_{l}}\right)\right)\left(\frac{\partial}{\partial t_{i}}, \frac{\partial}{\partial t_{j}}\right),
\end{aligned}
$$

where $R$ is the curvature operator of the Siegel metric on $\mathscr{T}_{g}$, while $\widetilde{R}$ is the one 
on $\mathscr{H}_{g}$. Set

Thus

$$
\Sigma_{i \bar{j} k \bar{l}}=\left(\sigma\left(\frac{\partial}{\partial t_{k}}\right), \sigma\left(\frac{\partial}{\partial t_{l}}\right)\right)\left(\frac{\partial}{\partial t_{i}}, \frac{\partial}{\partial t_{j}}\right) .
$$

$$
R_{i \bar{j} k \bar{l}}=\widetilde{R}_{i \bar{j} k \bar{l}}-\Sigma_{i \bar{j} k \bar{l}} .
$$

Thus we focus on the Siegel metric $\widetilde{\omega}_{s}$ and its curvature, and use the bounded domain $\mathscr{B}_{g}$ to simplify the computation.

Siegel geometry on $\mathscr{B}_{\mathrm{g}}$.

Theorem 5.1. The Siegel metric $\widetilde{\omega}_{s}$ on $\mathscr{B}_{g}$ has the full expansion formula

$$
\widetilde{\omega}_{s}=\frac{\mathrm{i}}{2} \sum_{n \geq 0} \sum_{\alpha \leq \beta} \sum_{\gamma \leq \delta} \frac{1}{n+1} \operatorname{tr}\left(S_{(\alpha \beta) \overline{(\gamma \delta)}}(W \bar{W})^{n+1}\right) d W_{\alpha \beta} \wedge d \overline{W_{\gamma \delta}} .
$$

Proof. Because the Siegel metric equals the Bergman metric, we use the Bergman kernel

$$
\begin{aligned}
\widetilde{\omega}_{s} & =-\frac{\mathrm{i}}{2} \partial \bar{\partial} \log \operatorname{det}\left(\mathbb{1}_{g}-\bar{W} W\right)=-\frac{\mathrm{i}}{2} \partial \bar{\partial} \operatorname{tr} \log \left(\mathbb{1}_{g}-\bar{W} W\right) \\
& =\frac{\mathrm{i}}{2} \partial \bar{\partial}\left(\sum_{n=1}^{\infty} \frac{1}{n} \operatorname{tr}(\bar{W} W)^{n}\right)=\frac{\mathrm{i}}{2} \partial \bar{\partial}\left(\sum_{n=1}^{\infty} \frac{1}{n} \operatorname{tr}(W \bar{W})^{n}\right) .
\end{aligned}
$$

Now $\widetilde{\omega}_{s}$ can be written as

$$
\widetilde{\omega}_{s}=\frac{\mathrm{i}}{2} \sum_{n \geq 0} \sum_{\alpha \leq \beta} \sum_{\gamma \leq \delta} \frac{1}{n+1} \operatorname{tr}\left(S_{(\alpha \beta) \overline{(\gamma \delta)}}(W \bar{W})^{n+1}\right) d W_{\alpha \beta} \wedge d \overline{W_{\gamma \delta}},
$$

where $S_{(\alpha \beta) \overline{(\gamma \delta)}}$ indicates taking derivatives along $\partial / \partial W_{\alpha \beta}$ and $\partial / \partial \overline{W_{\gamma \delta}}$ with $\alpha \leq$ $\beta, \gamma \leq \delta$ according to Definition 4.1. Since $W$ is symmetric, $\partial / \partial W_{\alpha \beta}$ takes the derivative with respect to $W_{\alpha \beta}$ and $W_{\beta \alpha}$.

Similarly, if we write $\widetilde{\omega}_{s}=\sum_{\alpha \leq \beta} \sum_{\gamma \leq \delta} \frac{\mathrm{i}}{2} \widetilde{\omega}_{(\alpha \beta) \overline{(\gamma \delta)}} d W_{\alpha \beta} \wedge d \overline{W_{\gamma \delta}}$, it is easy to see that

$$
\widetilde{\omega}_{(\alpha \beta) \overline{(\gamma \delta)}}(0)= \begin{cases}1 & \text { for } \alpha=\gamma=\beta=\delta, \\ 2 & \text { for } \alpha=\gamma \neq \beta=\delta, \\ 0 & \text { otherwise }\end{cases}
$$

and

$$
\widetilde{\omega}_{(\alpha \beta) \overline{(\gamma \delta)}}=\frac{\mathrm{i}}{2} \sum_{n \geq 0} \frac{1}{n+1} \operatorname{tr}\left(S_{(\alpha \beta) \overline{(\gamma \delta)}}(W \bar{W})^{n+1}\right) .
$$

In the following computation, the matrix $D$ is defined as

$$
D_{\alpha \beta}= \begin{cases}1 & \text { for } \alpha=\beta, \\ \sqrt{2} & \text { for } \alpha \neq \beta .\end{cases}
$$


Lemma 5.2. We have

$\widetilde{\omega}^{(\alpha \beta)}(\gamma \delta)=\frac{1}{D_{\alpha \beta}^{2}} \frac{1}{D_{\gamma \delta}^{2}} \widetilde{\omega}_{(\alpha \beta) \overline{(\gamma \delta)}}(0)$

$+\sum_{k \geq 1} \sum_{\substack{m_{i}>0,1 \leq i \leq l \\ m_{1}+\cdots+m_{l}=k}}(-1)^{l} \frac{1}{D_{\alpha \beta}^{2}} \frac{1}{D_{\gamma \delta}^{2}}\left(\frac{1}{m_{1}+1} \operatorname{tr}\left(S_{(\alpha \beta) \overline{\left(\alpha_{1} \beta_{1}\right)}}(W \bar{W})^{m_{1}+1}\right)\right)$

$\times\left(\frac{1}{m_{2}+1} \operatorname{tr}\left(S_{\left(\alpha_{1} \beta_{1}\right) \overline{\left(\alpha_{2} \beta_{2}\right)}}(W \bar{W})^{m_{2}+1}\right)\right) \cdots\left(\frac{1}{m_{l}+1} \operatorname{tr}\left(S_{\left(\alpha_{l-1} \beta_{l-1}\right)} \overline{(\gamma \delta)}(W \bar{W})^{m_{l}+1}\right)\right)$,

where $\alpha_{1}, \beta_{1}, \ldots, \alpha_{l-1}, \beta_{l-1}$ are free indices from 1 to $g$. (Here $\left(\alpha_{i} \beta_{i}\right)$ means taking the derivative just with respect to $W_{\alpha_{i} \beta_{i}}$; this convention will remain in force later.)

Proof. We use another coordinate $Y_{\alpha \beta}:=D_{\alpha \beta} X_{\alpha \beta}$ on $\mathscr{B}_{g}$ to rewrite $\widetilde{\omega}_{s}$. It is easy to check that $\widetilde{\omega}_{s}=\frac{i}{2} \sum_{\alpha \leq \beta} \sum_{\gamma \leq \delta} \widetilde{\Omega}_{(\alpha \beta)} \overline{(\gamma \delta)} d Y_{\alpha \beta} \wedge d \overline{Y_{\gamma \delta}}$, where

$$
\widetilde{\Omega}_{(\alpha \beta) \overline{(\gamma \delta)}}=\frac{1}{D_{\alpha \beta}} \frac{1}{D_{\gamma \delta}} \widetilde{\omega}_{(\alpha \beta) \overline{(\gamma \delta)}}
$$

and $\widetilde{\Omega}_{(\alpha \beta) \overline{(\gamma \delta)}}(0)=\delta_{\alpha \gamma} \delta_{\delta \beta}$ (Kronecker symbol). Now an application of Lemma 4.3 to $\widetilde{\Omega}_{(\alpha \beta) \overline{(\gamma \delta)}}$ yields

$$
\begin{array}{r}
\widetilde{\Omega}^{\overline{(\alpha \beta)}(\gamma \delta)}=\widetilde{\Omega}_{(\alpha \beta) \overline{(\gamma \delta)}}(0)+\sum_{k \geq 1} \sum_{\substack{m_{i}>0,1 \leq i \leq n \\
m_{1}+\cdots+m_{l}=k}} \sum_{\alpha_{1} \leq \beta_{1}} \ldots \sum_{\alpha_{l-1} \leq \beta_{l-1}}(-1)^{l}\left(\widetilde{\Omega}_{(\alpha \beta) \overline{\left(\alpha_{1} \beta_{1}\right)}}\right)_{m_{1}} \ldots \\
\times\left(\widetilde{\Omega}_{\left(\alpha_{l-1} \beta_{l-1}\right) \overline{(\gamma \delta)}}\right)_{m_{l}} .
\end{array}
$$

From (5-3) and the equality $\widetilde{\Omega}^{\overline{(\alpha \beta)}(\gamma \delta)}=D_{\alpha \beta} D_{\gamma \delta} \widetilde{\omega}^{\overline{(\alpha \beta)}(\gamma \delta)}$, we get the result.

Theorem 5.3. The curvature $\widetilde{\mathrm{R}}_{(\alpha \beta)} \overline{(\gamma \delta)(\zeta \eta)} \overline{(\sigma \tau)}$ is given by

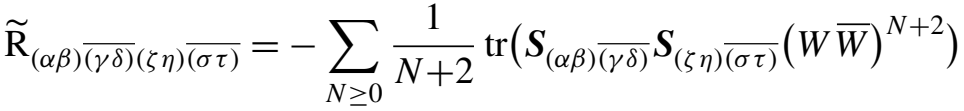

$$
\begin{aligned}
& +\sum_{N \geq 0} \sum_{\substack{N_{1} \geq 0, N_{2}>0, N_{3}>0 \\
\sum_{i=1}^{3} N_{i}=N+1}}\left[\sum_{\substack{m_{i}>0,1 \leq i \leq l \\
m_{1}+\cdots+m_{l}=N_{1}}}(-1)^{l}\left(\frac{1}{m_{1}+1} \operatorname{tr}\left(S_{(\lambda \mu) \overline{\left(\alpha_{1} \beta_{1}\right)}}(W \bar{W})^{m_{1}+1}\right)\right)\right. \\
& \left.\times\left(\frac{1}{m_{2}+1} \operatorname{tr}\left(S_{\left(\alpha_{1} \beta_{1}\right) \overline{\left(\alpha_{2} \beta_{2}\right)}}(W \bar{W})^{m_{2}+1}\right)\right) \cdots\left(\frac{1}{m_{l}+1} \operatorname{tr}\left(S_{\left(\alpha_{l-1} \beta_{l-1}\right) \overline{(\phi \psi)}}(W \bar{W})^{m_{l}+1}\right)\right)\right] \\
& \times \frac{1}{N_{2}+1} \operatorname{tr}\left(\boldsymbol{S}_{(\alpha \beta)} \boldsymbol{S}_{(\zeta \eta) \overline{(\lambda \mu)}}(W \bar{W})^{N_{2}+1}\right) \frac{1}{N_{3}+1} \operatorname{tr}\left(\boldsymbol{S}_{\overline{(\gamma \delta)}} \boldsymbol{S}_{(\phi \psi) \overline{(\sigma \tau)}}(W \bar{W})^{N_{3}+1}\right),
\end{aligned}
$$

where $\alpha_{1}, \beta_{1}, \ldots, \alpha_{l-1}, \beta_{l-1}, \lambda, \mu, \phi, \psi$ are free indices from 1 to $g$. (We use the convention that the quantity in square brackets equals $\delta_{\lambda \phi} \delta_{\mu \psi}$ if $N_{1}=0$.) 
Proof. We resort to the curvature formula again:

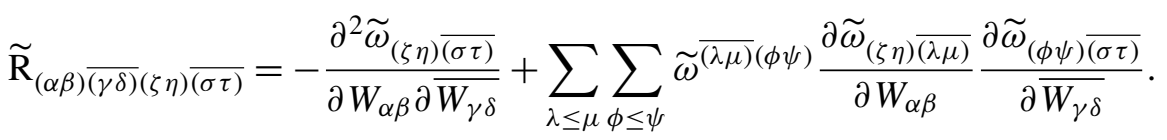

Also from (5-2), we have

$$
\begin{aligned}
\frac{\partial^{2} \widetilde{\omega}_{(\zeta \eta) \overline{(\sigma \tau)}}}{\partial X_{\alpha \beta} \partial \overline{X_{\gamma \delta}}} & =\sum_{k \geq 0} \frac{1}{k+2} \operatorname{tr}\left(\boldsymbol{S}_{(\alpha \beta) \overline{(\gamma \delta)}} \boldsymbol{S}_{(\zeta \eta) \overline{(\sigma \tau)}}(W \bar{W})^{k+2}\right), \\
\frac{\partial \widetilde{\omega}_{(\zeta \eta) \overline{(\lambda \mu)}}}{\partial X_{\alpha \beta}} & =\sum_{k \geq 1} \frac{1}{k+1} \operatorname{tr}\left(\boldsymbol{S}_{(\alpha \beta)} \boldsymbol{S}_{(\zeta \eta) \overline{(\lambda \mu)}}(W \bar{W})^{k+1}\right), \\
\frac{\partial \widetilde{\omega}_{(\phi \psi) \overline{(\sigma \tau)}}}{\partial \overline{X_{\gamma \delta}}} & =\sum_{k \geq 1} \frac{1}{k+1} \operatorname{tr}\left(\boldsymbol{S}_{\overline{(\gamma \delta)}} \boldsymbol{S}_{(\phi \psi) \overline{(\sigma \tau)}}(W \bar{W})^{k+1}\right) .
\end{aligned}
$$

From this and Lemma 5.2, the result follows.

Based on Theorem 5.3, the holomorphic sectional curvature $\mathrm{H}(V)$ of $\widetilde{\omega}_{s}$ along the direction $V=\sum_{\alpha \leq \beta} V_{\alpha \beta} \partial / \partial W_{\alpha \beta}$ at the zero matrix of $\mathscr{B}_{g}$ can be easily gotten:

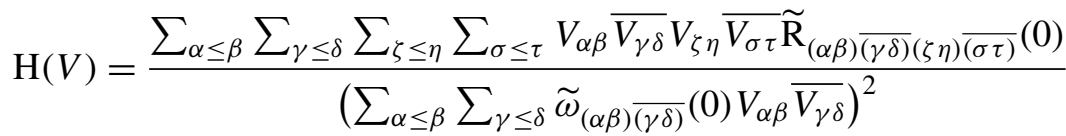

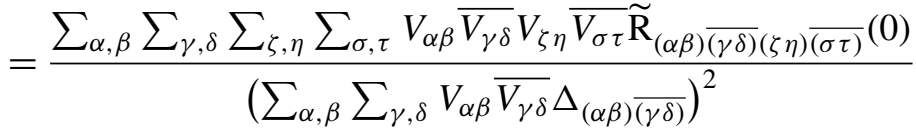

$$
\begin{aligned}
& =-2 \frac{\sum_{\alpha, \beta, \gamma, \delta} V_{\alpha \beta} \overline{V_{\beta \gamma}} V_{\gamma \delta} \overline{V_{\delta \alpha}}}{\left(\sum_{\alpha, \beta} V_{\alpha \beta} \overline{V_{\alpha \beta}}\right)^{2}} \text {. }
\end{aligned}
$$

In the second equality, $V_{\alpha \beta}$ has a symmetric extension to the whole matrix, and there $(\alpha \beta)$ in $\widetilde{\mathrm{R}}_{(\alpha \beta) \overline{(\gamma \delta)}(\zeta \eta) \overline{(\sigma \tau)}}$ stands for the derivative with respect to $W_{\alpha \beta}$, not to both $W_{\alpha \beta}$ and $W_{\beta \alpha}$.

By the mean value inequality,

$$
\frac{1}{g} \leq \frac{\sum_{\alpha, \beta, \gamma, \delta} V_{\alpha \beta} \overline{V_{\beta \gamma}} V_{\gamma \delta} \overline{V_{\delta \alpha}}}{\left(\sum_{\alpha, \beta} V_{\alpha \beta} \overline{V_{\alpha \beta}}\right)^{2}} \leq 1
$$

Thus

$$
-2 \leq \mathrm{H}(V) \leq-\frac{2}{g} .
$$

Yin [2010, Corollaries 1.1 and 1.2 of Chapter 4] has reproved the classical fact that $\mathrm{H}(V)=-2$ if and only if $V$ is a symmetric matrix of rank 1 . 
Proposition 5.4. If $\mathrm{H}(V)=-2 / g$, then $V=k U U^{T}$ with $k>0$ and $U \in \mathrm{M}(g, \mathbb{C})$ unitary.

Proof. $\mathrm{H}(V)=-2 / g$ forces the following inequalities to become equalities:

$$
\begin{aligned}
\frac{\sum_{\alpha, \beta, \gamma, \delta} V_{\alpha \beta} \overline{V_{\beta \gamma}} V_{\gamma \delta} \overline{V_{\delta \alpha}}}{\left(\sum_{\alpha, \beta} V_{\alpha \beta} \overline{V_{\alpha \beta}}\right)^{2}} & =\frac{\sum_{\alpha, \gamma}\left|\sum_{\beta} V_{\alpha \beta} \overline{V_{\gamma \beta}}\right|^{2}}{\left(\sum_{\alpha, \beta}\left|V_{\alpha \beta}\right|^{2}\right)^{2}} \\
& =\frac{\sum_{\alpha=\gamma}\left|\sum_{\beta} V_{\alpha \beta} \overline{V_{\gamma \beta}}\right|^{2}+\sum_{\alpha \neq \gamma}\left|\sum_{\beta} V_{\alpha \beta} \overline{V_{\gamma \beta}}\right|^{2}}{\left(\sum_{\alpha, \beta}\left|V_{\alpha \beta}\right|^{2}\right)^{2}} \\
& \geq \frac{\left.\left.\sum_{\alpha}\left|\sum_{\beta}\right| V_{\alpha \beta}\right|^{2}\right|^{2}}{\left(\sum_{\alpha, \beta}\left|V_{\alpha \beta}\right|^{2}\right)^{2}} \geq \frac{(1 / g)\left(\sum_{\alpha, \beta}\left|V_{\alpha \beta}\right|^{2}\right)^{2}}{\left(\sum_{\alpha, \beta}\left|V_{\alpha \beta}\right|^{2}\right)^{2}} .
\end{aligned}
$$

This is equivalent to $\sum_{\beta} V_{\alpha \beta} \overline{V_{\gamma \beta}}=0$ and $\sum_{\beta}\left|V_{\alpha \beta}\right|^{2}=\sum_{\beta}\left|V_{\gamma \beta}\right|^{2}$ for any $\alpha \neq \gamma$. Up to a constant multiple that is a real number bigger than zero, $V_{\alpha \beta}$ is symmetric and unitary. Here is a result from [Mok 1989, p. 70]: If $V \in \mathbf{M}(g, \mathbb{C})$ is complex symmetric, $V$ can be written as

$$
U^{T}\left(\begin{array}{llll}
\lambda_{1} & & & \\
& \lambda_{2} & & \\
& & \ddots & \\
& & & \lambda_{g}
\end{array}\right) U,
$$

with $U$ unitary and $\lambda_{i} \geq 0$. Also since $V$ is unitary,

$$
U^{T}\left(\begin{array}{cccc}
\lambda_{1}^{2} & & & \\
& \lambda_{2}^{2} & & \\
& & \ddots & \\
& & & \lambda_{g}^{2}
\end{array}\right) \bar{U}=\mathbb{1}_{g},
$$

which is equivalent to $\lambda_{i}^{2}=1$, so $\lambda_{i}=1$. Thus $V=k U U^{T}$ with $k>0$ and $U$ unitary.

Yin [2010, Lemma 1.2 and Remark 1.6 of Chapter 4] showed that $\widetilde{R}$ is very strongly seminegative in the sense of Siu, but not very strongly negative.

Remark 5.5. In the literature one sees $\mathrm{H}(V) \in[-1,-1 / g]$, since the Bergmann kernel used is $\rho=-2 \log \operatorname{det}\left(\mathbb{1}_{g}-\overline{W^{T}} W\right)$.

The second fundamental form and the totally geodesic submanifold. Recall that

$$
\mathscr{T}_{\text {or }} \stackrel{\text { glor }^{\text {t }}}{\longrightarrow} \mathscr{H}_{g} \longrightarrow \mathscr{B}_{g} .
$$


The period map $g^{\text {tor }}$ is given by, from (2-7),

$$
g^{\text {tor }}(t)=\left(\mathrm{i} \mathbb{1}_{g}-\mathrm{i} A(t)^{T}\right)\left(\mathbb{1}_{g}+A(t)^{T}\right)^{-1}=\mathrm{i} \frac{\mathbb{1}_{g}-A(t)^{T}}{\mathbb{1}_{g}+A(t)^{T}},
$$

where we normalize the target point to $i \mathbb{1}_{g}$. The transformation from $\mathscr{H}_{g}$ to $\mathscr{B}_{g}$ is given by

$$
W=\frac{\mathbb{1}_{g}+\mathrm{i} Z}{\mathbb{1}_{g}-\mathrm{i} Z} .
$$

Hence $W=A(t)^{T}=A(t)$, where $A(t)$ is symmetric.

Together with Theorem 5.3, we have

$$
\begin{aligned}
& \widetilde{\mathrm{R}}_{i \bar{j} k \bar{l}}=\sum_{\alpha \leq \beta} \sum_{\gamma \leq \delta} \sum_{\zeta \leq \eta} \sum_{\sigma \leq \tau} \widetilde{\mathrm{R}}_{(\alpha \beta) \overline{(\gamma \delta)(\zeta \eta) \overline{(\sigma \tau)}}} \frac{\partial W_{\alpha \beta}}{\partial t_{i}} \frac{\overline{\partial W_{\gamma \delta}}}{\partial t_{j}} \frac{\partial W_{\zeta \eta}}{\partial t_{k}} \frac{\overline{\partial W_{\sigma \tau}}}{\partial t_{l}} \\
& =\sum_{\alpha, \beta} \sum_{\gamma, \delta} \sum_{\zeta, \eta} \sum_{\sigma, \tau} \widetilde{\mathrm{R}}_{(\alpha \beta) \overline{(\gamma \delta)(\zeta \eta) \overline{(\sigma \tau)}}} \frac{\partial W_{\alpha \beta}}{\partial t_{i}} \frac{\overline{\partial W_{\gamma \delta}}}{\partial t_{j}} \frac{\partial W_{\zeta \eta}}{\partial t_{k}} \frac{\overline{\partial W_{\sigma \tau}}}{\partial t_{l}} \\
& =-\sum_{N \geq 0} \frac{1}{N+2} \operatorname{tr}\left(S_{i \bar{j}}^{\prime} S_{k \bar{l}}^{\prime}(W \bar{W})^{N+2}\right) \\
& +\sum_{N \geq 0} \sum_{\substack{N_{1} \geq 0, N_{2}>0, N_{3}>0 \\
\sum_{i=1}^{3} N_{i}=N+1}}\left[\sum_{\substack{m_{i}>0,1 \leq i \leq l \\
m_{1}+\cdots+m_{l}=N_{1}}}(-1)^{l}\right. \\
& \times\left(\frac{1}{m_{1}+1} \operatorname{tr}\left(S_{(\lambda \mu) \overline{\left(\alpha_{1} \beta_{1}\right)}}(W \bar{W})^{m_{1}+1}\right)\right) \\
& \times\left(\frac{1}{m_{2}+1} \operatorname{tr}\left(S_{\left(\alpha_{1} \beta_{1}\right) \overline{\left(\alpha_{2} \beta_{2}\right)}}(W \bar{W})^{m_{2}+1}\right)\right) \ldots \\
& \left.\times\left(\frac{1}{m_{l}+1} \operatorname{tr}\left(S_{\left(\alpha_{l-1} \beta_{l-1}\right) \overline{(\phi \psi)}}(W \bar{W})^{m_{l}+1}\right)\right)\right] \\
& \times\left[\frac{1}{N_{2}+1} \operatorname{tr}\left(S_{i}^{\prime} S_{k(\lambda \mu)}^{\prime}(W \bar{W})^{N_{2}+1}\right)\right] \\
& \times\left[\frac{1}{N_{3}+1} \operatorname{tr}\left(S_{\bar{j}}^{\prime} S_{(\phi \psi) \bar{l}}^{\prime}(W \bar{W})^{N_{3}+1}\right)\right] .
\end{aligned}
$$

In the second equality, we also need a symmetric extension of the indices, as in (5-4). In the third equality, $S_{i}$ means taking the derivative along $\partial / \partial t_{i}$, since $W=A(t)$ can be seen as a matrix with variable $t$; and $\partial / \partial t_{i}$ and $\partial / \partial t_{k}$ still run through all $W$ 's but can't operate simultaneously on a single $W$, according to Definition 4.1. 
Since the calculation is a little bit complicated, we will present a more detailed formula in the Appendix. Also, the second fundamental form of $g^{\text {tor }}$, restricted to a nonhyperelliptic locus, can be computed out. However, it is difficult to understand the vanishing of the second fundamental form entirely. Partial results are given as follows.

Proposition 5.6. The second fundamental form of the nonhyperelliptic locus at the central point is

$$
\Sigma_{i \bar{j} k \bar{l}}(0)=\operatorname{tr}\left(A_{i k} \overline{A_{j l}}\right) .
$$

Proof. From (5-5), we easily have $\widetilde{\mathrm{R}}_{i \bar{j} k \bar{l}}(0)=-\frac{1}{2} \operatorname{tr}\left(S_{i \bar{j}}^{\prime} S_{k \bar{l}}^{\prime}\left(A_{1}(t) \overline{A_{1}(t)} A_{1}(t) \overline{A_{1}(t)}\right)\right)$. Also, Theorem 4.4 tells us

$$
\begin{aligned}
\mathrm{R}_{i \bar{j} k \bar{l}}(0)=-\operatorname{tr}\left(S_{i \bar{j}} S_{k \bar{l}}\left(A_{2}(t) \overline{A_{2}(t)}\right)\right)-\frac{1}{2} \operatorname{tr}\left(S_{i \bar{j}} S_{k \bar{l}}\left(A_{1}(t) \overline{A_{1}(t)} A_{1}(t) \overline{A_{1}(t)}\right)\right) \\
+\left[\operatorname{tr}\left(S_{i} S_{k \bar{p}}\left(A_{2}(t) \overline{A_{1}(t)}\right)\right)\right]\left[\operatorname{tr}\left(S_{\bar{j}} S_{p \bar{l}}\left(A_{1}(t) \overline{A_{2}(t)}\right)\right)\right] .
\end{aligned}
$$

Hence $\Sigma_{i \bar{j} k l}(0)=\operatorname{tr}\left(A_{i k} \overline{A_{j l}}\right)-\operatorname{tr}\left(A_{i k} \overline{A_{p}}\right) \operatorname{tr}\left(A_{p} \overline{A_{j l}}\right)$. By the use of (4-5), this proposition follows easily.

Corollary 5.7. Holomorphic sectional curvature along a totally geodesic submanifold $M$ in a nonhyperelliptic locus of $\mathscr{T}_{g}$ is bounded from below.

This shows indicates that a totally geodesic submanifold can't be arbitrarily negatively curved.

Proof. Proposition 5.6 tells us that $A_{i j}=0$ on the totally geodesic manifold $M$. From (4-7) and (4-8), the holomorphic sectional curvature $\mathrm{H}(v)$ becomes

$$
\mathrm{H}(v)=-2 \frac{\operatorname{tr}\left(\left(a_{i} A_{i}\right) \overline{\left(a_{j} A_{j}\right)}\left(a_{k} A_{k}\right) \overline{\left(a_{l} A_{l}\right)}\right)}{\left(\operatorname{tr}\left(\left(a_{i} A_{i}\right) \overline{\left(a_{j} A_{j}\right)}\right)\right)^{2}} .
$$

By (4-9),

$$
\mathrm{H}(v) \geq-2 .
$$

Proposition 5.8. The totally geodesic manifold $M$ in the nonhyperelliptic locus of $\mathscr{T}_{g}$ must be locally symmetric.

Proof. We just need to check that $\nabla \mathrm{R}=0$ on $M$. We use the well known formulas

$$
\begin{aligned}
& \nabla_{p} \mathbf{R}_{i \bar{j} k \bar{l}}=\frac{\partial}{\partial t_{p}} \mathbf{R}_{i \bar{j} k \bar{l}}-\Gamma_{p i}^{q} \mathbf{R}_{q \bar{j} k \bar{l}}-\Gamma_{p k}^{q} \mathbf{R}_{i \bar{j} q \bar{l}}, \\
& \nabla_{\bar{p}} \mathbf{R}_{i \bar{j} k \bar{l}}=\frac{\partial}{\partial \bar{t}_{p}} \mathbf{R}_{i \bar{j} k \bar{l}}-\overline{\Gamma_{p j}^{q}} \mathbf{R}_{i \bar{q} k \bar{l}}-\overline{\Gamma_{p l}^{q}} \mathbf{R}_{i \bar{j} k \bar{q}} .
\end{aligned}
$$

The normal coordinate gives us $\Gamma_{i j}^{k}(0)=0$, and thus both $\nabla_{p} \mathrm{R}_{i \bar{j} k \bar{l}}(0)$ and $\nabla_{\bar{p}} \mathrm{R}_{i \bar{j} k l}(0)$ concern the first derivative of $\mathrm{R}_{i j \bar{k} \bar{l}}$. By Proposition 5.6, $A_{i j}=0$, i.e., $A_{2}(t)=0$, follows from the fact that the manifold $M$ is totally geodesic. Also, Theorem 4.4 
implies that

$$
\begin{aligned}
\mathrm{R}_{i \bar{j} k \bar{l}}^{(1)}= & -\operatorname{tr}\left(S_{i \bar{j}} S_{k \bar{l}}\left(A_{2}(t) \overline{A_{3}(t)}+A_{3}(t) \overline{A_{2}(t)}\right)\right) \\
& -\operatorname{tr}\left(S_{i j} S_{k \bar{l}}\left(A_{2}(t) \overline{A_{1}(t)} A_{1}(t) \overline{A_{1}(t)}+A_{1}(t) \overline{A_{2}(t)} A_{1}(t) \overline{A_{1}(t)}\right)\right) \\
& +\operatorname{tr}\left(S_{i} S_{k \bar{p}}\left(\frac{1}{2} A_{1}(t) \overline{A_{1}(t)} A_{1}(t) \overline{A_{1}(t)}+A_{3}(t) \overline{A_{1}(t)}\right)\right) \operatorname{tr}\left(S_{\bar{j}} S_{p \bar{l}}\left(A_{1}(t) \overline{A_{2}(t)}\right)\right) \\
& +\operatorname{tr}\left(S_{i} S_{k \bar{p}}\left(A_{2}(t) \overline{A_{1}(t)}\right)\right) \operatorname{tr}\left(S_{\bar{j}} S_{p \bar{l}}\left(\frac{1}{2} A_{1}(t) \overline{A_{1}(t)} A_{1}(t) \overline{A_{1}(t)}+A_{1}(t) \overline{A_{3}(t)}\right)\right) \\
& -\operatorname{tr}\left(S_{q \bar{p}}\left(A_{2}(t) \overline{A_{1}(t)}+A_{1}(t) \overline{A_{2}(t)}\right)\right) \\
& \quad \operatorname{tr}\left(S_{i} S_{k \bar{q}}\left(A_{2}(t) \overline{A_{1}(t)}\right)\right) \operatorname{tr}\left(S_{\bar{j}} S_{p \bar{l}}\left(A_{1}(t) \overline{A_{2}(t)}\right)\right) .
\end{aligned}
$$

Every summand above has an $A_{2}(t)$ term. So $\mathbf{R}_{i \bar{j} k \bar{l}}^{(1)}=0$, and thus $\nabla_{p} \mathbf{R}_{i \bar{j} k \bar{l}}(0)=0$ and $\nabla_{\bar{p}} \mathrm{R}_{i j \bar{k} k}(0)=0$.

\section{Appendix}

We give here the full formula for the second fundamental form. Let $E_{\beta}^{\alpha}$ be defined by $\left(E_{\beta}^{\alpha}\right)_{\gamma \delta}=\delta_{\alpha \gamma} \delta_{\beta \delta}$ (Kronecker delta). The following example will serve to illustrate the meaning of the symbol $\boldsymbol{P}$ (permutation summation):

$\boldsymbol{P}(A \bar{B} C \bar{D} E \bar{F} G \bar{H})$

$$
=A \bar{B} C \bar{D} E \bar{F} G \bar{H}+C \bar{B} A \bar{D} E \bar{F} G \bar{H}+E \bar{B} C \bar{D} A \bar{F} G \bar{H}+G \bar{B} C \bar{D} E \bar{F} A \bar{H},
$$

where $A$ to $H$ are all complex matrices. The matrices with bars are stationary, and those with bars travel through those without bars.

Replace $W$ by $A(t)$ in (5-5) and rearrange that formula according to degrees in $t$ to get

$$
\begin{aligned}
& \widetilde{\mathrm{R}}_{i \bar{j} k \bar{l}}=-\sum_{\substack{N \geq 0 \\
m_{i}>0,1 \leq i \leq 2 l, l \geq 2 \\
m_{1}+\cdots+m_{2 l}=N+4}} \frac{1}{l} \operatorname{tr}\left(S_{i j}^{\prime} S_{k \bar{l}}^{\prime}\left(A_{m_{1}}(t) \ldots \overline{A_{m_{2 l}}(t)}\right)\right) \\
& +\sum_{\substack{N_{1} \geq 0, N_{2}>0, N_{3}>0 \\
\sum_{i=1}^{3} N_{i}=N}}\left[\sum_{\substack{m_{i}>0,1 \leq i \leq l \\
m_{1}+\cdots+m_{l}=N_{1}}}(-1)^{l}\right. \\
& \times \sum_{\substack{m_{1 n}>0, \sum_{n=1}^{2 s_{1}} m_{1 n}=m_{1}}} \operatorname{tr}\left(\boldsymbol{P}\left(E_{\mu}^{\lambda} \overline{A_{m_{11}}(t)} \ldots A_{m_{12 s_{1}}}(t) \overline{E_{\beta_{1}}^{\alpha_{1}}}\right)\right) \\
& \times \sum_{\substack{m_{2 n}>0, \sum_{n=1}^{2 s_{2}} m_{2 n}=m_{2}}} \operatorname{tr}\left(\boldsymbol{P}\left(E_{\beta_{1}}^{\alpha_{1}} \overline{A_{m_{21}}(t)} \ldots A_{m_{22 s_{2}}}(t) \overline{E_{\beta_{2}}^{\alpha_{2}}}\right)\right) \ldots \\
& \left.\times \sum_{\substack{m_{l n}>0, \sum_{n=1}^{2 s_{l}} m_{l n}=m_{l}}} \operatorname{tr}\left(\boldsymbol{P}\left(E_{\beta_{l-1}}^{\alpha_{l-1}} \overline{A_{m_{l 1}}(t)} \ldots A_{m_{l 2 s_{l}}}(t) \overline{E_{\psi}^{\phi}}\right)\right)\right]
\end{aligned}
$$




$$
\begin{aligned}
& \times\left[\sum_{\substack{m_{i}>0, \sum_{i=1}^{2+1} m_{i}=N_{2}+2}} \operatorname{tr}\left(S_{i}^{\prime} S_{k}^{\prime}\left(A_{m_{1}}(t) \ldots A_{m_{2 l+1}}(t) \overline{E_{\mu}^{\lambda}}\right)\right)\right] \\
& \times\left[\sum_{\substack{m_{i}>0, \sum_{i=1}^{2 l+1} m_{i}=N_{3}+2}} \operatorname{tr}\left(S_{\bar{j}}^{\prime} S_{\bar{l}}^{\prime}\left(E_{\psi}^{\phi} \overline{A_{m_{1}}(t)} \ldots \overline{A_{m_{2 l+1}}(t)}\right)\right)\right] .
\end{aligned}
$$

By convention, the term in the first square brackets has the value $\delta_{\lambda \phi} \delta_{\mu \psi}$ if $N_{1}=0$.

Theorem A.9. The second fundamental form $\Sigma_{i \bar{j} k \bar{l}}$ of the nonhyperelliptic locus is

$$
\begin{aligned}
& \Sigma_{i \bar{j} k \bar{l}} \\
& =\sum_{N \geq 0} \frac{1}{l}\left(\sum_{m_{i}>0,1 \leq i \leq 2 l} \operatorname{tr}\left(S_{i j} S_{k \bar{l}}\left(A_{m_{1}}(t) \overline{A_{m_{2}}(t)} \ldots \overline{A_{m_{2 l}}(t)}\right)\right)\right. \\
& \sum_{i=1}^{2 l} m_{i}=N+4 \\
& \left.-\sum_{m_{i}>0, l \geq 2} \operatorname{tr}\left(S_{i \bar{j}}^{\prime} S_{k \bar{l}}^{\prime}\left(A_{m_{1}}(t) \ldots \overline{A_{m_{2 l}}(t)}\right)\right)\right) \\
& \sum_{i=1}^{2 l} m_{i}=N+4 \\
& +\sum_{N \geq 0} \sum_{\substack{N_{1} \geq 0, N_{2}>0, N_{3}>0 \\
\sum_{i=1}^{3} N_{i}=N}}\left[\sum_{\substack{m_{i}>0,1 \leq i \leq l \\
m_{1}+\cdots+m_{l}=N_{1}}}(-1)^{l}\right.
\end{aligned}
$$

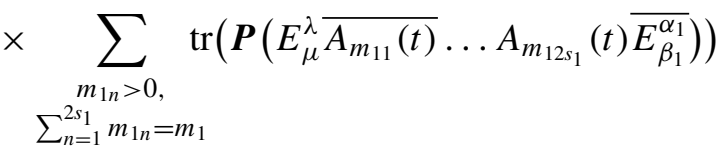

$$
\begin{aligned}
& \times \quad \sum_{m_{2 n}>0} \operatorname{tr}\left(\boldsymbol{P}\left(E_{\beta_{1}}^{\alpha_{1}} \overline{A_{m_{21}}(t)} \ldots A_{m_{22 s_{2}}}(t) \overline{E_{\beta_{2}}^{\alpha_{2}}}\right)\right) \ldots \\
& \sum_{n=1}^{2 s_{2}} m_{2 n}=m_{2} \\
& \left.\times \quad \sum_{m_{l n}>0} \operatorname{tr}\left(\boldsymbol{P}\left(E_{\beta_{l-1}}^{\alpha_{l-1}} \overline{A_{m_{l 1}}(t)} \ldots A_{m_{l s_{l}}}(t) \overline{E_{\psi}^{\phi}}\right)\right)\right] \\
& \sum_{n=1}^{2 s_{l}} m_{l n}=m_{l} \\
& \times\left[\sum_{\substack{m_{i}>0 \\
\sum_{i=1}^{2 l+1} m_{i}=N_{2}+2}} \operatorname{tr}\left(S_{i}^{\prime} S_{k}^{\prime}\left(A_{m_{1}}(t) \ldots A_{m_{2 l+1}}(t) \overline{E_{\mu}^{\lambda}}\right)\right)\right] \\
& \times\left[\sum_{\substack{m_{i}>0, \sum_{i=1}^{2 l+1} m_{i}=N_{3}+2}} \operatorname{tr}\left(S_{j}^{\prime} S_{\bar{l}}^{\prime}\left(E_{\psi}^{\phi} \overline{A_{m_{1}}(t)} \ldots \overline{A_{m_{2 l+1}}(t)}\right)\right)\right] \\
& -\sum_{N \geq 0} \sum_{\substack{N_{i} \geq 0,1 \leq i \leq 3 \\
\sum_{i=1}^{3}}}\left[\sum_{\substack{N_{i}=N \\
m_{i}>0,1 \leq i \leq l \\
m_{1}+\cdots+m_{l}=N_{1}}}(-1)^{l} \prod_{i=1}^{l} \frac{1}{s_{i}}\right.
\end{aligned}
$$




$$
\begin{aligned}
& \times \sum_{\substack{m_{1 n}>0,1 \leq n \leq 2 s_{1} \\
\sum_{n=1}^{2 s} 1}} \operatorname{tr}\left(S_{q \overline{i_{1}}}\left(A_{m_{11}}(t) \ldots \overline{A_{m_{12 s_{1}}}(t)}\right)\right) \\
& \times \sum_{\substack{m_{2 n}>0,1 \leq n \leq 2 s_{2} \\
\sum_{n=1}^{2 s_{2}} m_{2 n}=m_{2}+2}} \operatorname{tr}\left(S_{i_{1} \overline{i_{2}}}\left(A_{m_{21}}(t) \ldots \overline{A_{m_{22 s_{2}}}(t)}\right)\right) \ldots \\
& \left.\times \ldots \sum_{\substack{m_{l n}>0,1 \leq n \leq 2 s_{l} \\
\sum_{n=1}^{2 s_{l}} m_{l n}=m_{l}+2}} \operatorname{tr}\left(S_{i_{l-1} \bar{p}}\left(A_{m_{l 1}}(t) \ldots \overline{A_{m_{l 2} l}(t)}\right)\right)\right] \\
& \times\left[\sum_{\substack{m_{i}>0,1 \leq i \leq 2 l \\
\sum_{i=1}^{2 l} m_{i}=N_{2}+3}} \frac{1}{l} \operatorname{tr}\left(S_{i} S_{k \bar{q}}\left(A_{m_{1}}(t) \ldots \overline{A_{m_{2 l}}(t)}\right)\right)\right] \\
& \times\left[\sum_{\substack{m_{i}>0,1 \leq i \leq 2 l \\
\sum_{i=1}^{2 l} m_{i}=N_{3}+3}} \frac{1}{l} \operatorname{tr}\left(S_{\bar{j}} S_{p \bar{l}}\left(A_{m_{1}}(t) \ldots \overline{A_{m_{2 l}}(t)}\right)\right)\right] .
\end{aligned}
$$

Proof. This follows from Theorem 4.4, the formula for $\widetilde{\mathrm{R}}_{i \bar{j} k \bar{l}}$ above and (5-1).

\section{Acknowledgements}

The authors thank their advisor, Professor Kefeng Liu, for his constant help and encouragement. They are also grateful to Dr. Fangliang Yin, Professor Fangyang Zheng and Dr. Shengmao Zhu for their useful discussions and comments. The referees' useful suggestions and the editors' excellent work are also appreciated.

\section{References}

[Ahlfors 1966] L. V. Ahlfors, Lectures on quasiconformal mappings, Van Nostrand, Princeton, 1966. Reprinted Brooks/Cole 1987 and AMS 2006. MR 2009d:30001 Zbl 1103.30001

[Bojarski 1955] B. V. Bojarski, "Homeomorphic solutions of Beltrami systems", Dokl. Akad. Nauk SSSR (N.S.) 102 (1955), 661-664. In Russian. MR 17,157a

[Colombo and Frediani 2010] E. Colombo and P. Frediani, "Siegel metric and curvature of the moduli space of curves”, Trans. Amer. Math. Soc. 362:3 (2010), 1231-1246. MR 2010h:14046 Zbl 1196.14025

[Farb 2006] B. Farb (editor), Problems on mapping class groups and related topics, Proceedings of Symposia in Pure Mathematics 74, Amer. Math. Soc., Providence, RI, 2006. MR 2007e:57001 Zbl 1098.58001

[Farkas and Kra 1992] H. M. Farkas and I. Kra, Riemann surfaces, 2nd ed., Graduate Texts in Mathematics 71, Springer, New York, 1992. MR 93a:30047 Zbl 0764.30001

[Hain 1999] R. Hain, "Locally symmetric families of curves and Jacobians", pp. 91-108 in Moduli of curves and abelian varieties, edited by C. Faber and E. Looijenga, Aspects Math. E33, Vieweg, Braunschweig, 1999. MR 2000i:14040 Zbl 0966.14018 
[Liu et al. 2012a] K. F. Liu, S. Rao, and X. K. Yang, "Hodge theory and deformations of Kähler manifolds", preprint, 2012. arXiv 1207.1182

[Liu et al. 2012b] K. F. Liu, Q. T. Zhao, and S. Rao, "New proofs of the Torelli theorems for Riemann surfaces", preprint, 2012. arXiv 1207.5697

[Mayer 1969] A. Mayer, "Rauch's variational formula and the heat equation", Math. Ann. 181 (1969), 53-59. MR 40 \#1588 Zbl 0159.22403

[Mok 1989] N. Mok, Metric rigidity theorems on Hermitian locally symmetric manifolds, Series in Pure Mathematics 6, World Scientific, Teaneck, NJ, 1989. MR 92d:32046 Zbl 0912.32026

[Moonen 1995] B. J. J. Moonen, Special points and linearity properties of Shimura varieties, thesis, University of Utrecht, 1995.

[Moonen and Oort 2013] B. J. J. Moonen and F. Oort, "The Torelli locus and special subvarieties", pp. 549-594 in Handbook of moduli, vol. 2, edited by G. Farkas and I. Morrison, Advanced Lectures in Mathematics 25, International Press, Somerville, MA, 2013.

[Morrey 1938] C. B. Morrey, Jr., "On the solutions of quasi-linear elliptic partial differential equations", Trans. Amer. Math. Soc. 43:1 (1938), 126-166. MR 1501936 Zbl 0018.40501

[Oort 1997] F. Oort, "Canonical liftings and dense sets of CM-points", pp. 228-234 in Arithmetic geometry (Cortona, 1994), edited by F. Catanese, Sympos. Math. 37, Cambridge University Press, Cambridge, 1997. MR 98g:14056 Zbl 0911.14018

[Oort 2003] F. Oort, "Special points in Shimura varieties: an introduction", lecture notes, 2003, http:// www.staff.science.uu.nl/ oort0109/A-IC.SpecialPoints.ps.

[Rauch 1959] H. E. Rauch, "Weierstrass points, branch points, and moduli of Riemann surfaces", Comm. Pure Appl. Math. 12 (1959), 543-560. MR 22 \#1666 Zbl 0091.07301

[Toledo 1987] D. Toledo, "Nonexistence of certain closed complex geodesics in the moduli space of curves", Pacific J. Math. 129:1 (1987), 187-192. MR 88j:32026 Zbl 0648.14018

[Yin 2010] F. L. Yin, Geometry of period map of Riemann surface, thesis, Zhejiang University, 2010.

Received August 11, 2012.

QUANTING ZHAO

SCHOOL OF MATHEMATICS AND STATISTICS

Central China Normal University

WUHAN 430079

CHINA

zhaoquanting@126.com

SHENG RAO

SCHOOL OF MATHEMATICS AND STATISTICS

WUHAN UNIVERSITY

WUHAN 430072

CHINA

likeanyone@zju.edu.cn

Past affiliation of both authors:

Center of Mathematical Sciences

ZHEJIANG UNIVERSITY

HANGZHOU 310027

CHINA 


\title{
PACIFIC JOURNAL OF MATHEMATICS
}

\author{
msp.org/pjm
}

Founded in 1951 by E. F. Beckenbach (1906-1982) and F. Wolf (1904-1989)

\section{EDITORS}

V. S. Varadarajan (Managing Editor)

Department of Mathematics

University of California

Los Angeles, CA 90095-1555

pacific@math.ucla.edu

Paul Balmer

Department of Mathematics

University of California

Los Angeles, CA 90095-1555

balmer@math.ucla.edu

Daryl Cooper

Department of Mathematics

University of California

Santa Barbara, CA 93106-3080 cooper@math.ucsb.edu

Jiang-Hua $\mathrm{Lu}$

Department of Mathematics

Pokfulam Rd., Hong Kong jhlu@maths.hku.hk
The University of Hong Kong

Don Blasius

Department of Mathematics University of California

Los Angeles, CA 90095-1555

blasius@math.ucla.edu

Robert Finn

Department of Mathematics Stanford University

Stanford, CA 94305-2125

finn@math.stanford.edu

Sorin Popa

Department of Mathematics

University of California

Los Angeles, CA 90095-1555 popa@math.ucla.edu

Paul Yang

Department of Mathematics Princeton University

Princeton NJ 08544-1000

yang@math.princeton.edu

\section{PRODUCTION}

Silvio Levy, Scientific Editor, production@msp.org

\section{SUPPORTING INSTITUTIONS}

ACADEMIA SINICA, TAIPEI

CALIFORNIA INST. OF TECHNOLOGY

INST. DE MATEMÁTICA PURA E APLICADA

KEIO UNIVERSITY

MATH. SCIENCES RESEARCH INSTITUTE

NEW MEXICO STATE UNIV.

OREGON STATE UNIV.

\author{
STANFORD UNIVERSITY \\ UNIV. OF BRITISH COLUMBIA \\ UNIV. OF CALIFORNIA, BERKELEY \\ UNIV. OF CALIFORNIA, DAVIS \\ UNIV. OF CALIFORNIA, LOS ANGELES \\ UNIV. OF CALIFORNIA, RIVERSIDE \\ UNIV. OF CALIFORNIA, SAN DIEGO \\ UNIV. OF CALIF., SANTA BARBARA
}

\author{
Vyjayanthi Chari \\ Department of Mathematics \\ University of California \\ Riverside, CA 92521-0135 \\ chari@math.ucr.edu \\ Kefeng Liu \\ Department of Mathematics \\ University of California \\ Los Angeles, CA 90095-1555 \\ liu@math.ucla.edu \\ Jie Qing \\ Department of Mathematics \\ University of California \\ Santa Cruz, CA 95064 \\ qing@cats.ucsc.edu
}

These supporting institutions contribute to the cost of publication of this Journal, but they are not owners or publishers and have no responsibility for its contents or policies.

See inside back cover or msp.org/pjm for submission instructions.

The subscription price for 2013 is US \$400/year for the electronic version, and \$485/year for print and electronic.

Subscriptions, requests for back issues and changes of subscribers address should be sent to Pacific Journal of Mathematics, P.O. Box 4163, Berkeley, CA 94704-0163, U.S.A. The Pacific Journal of Mathematics is indexed by Mathematical Reviews, Zentralblatt MATH, PASCAL CNRS Index, Referativnyi Zhurnal, Current Mathematical Publications and the Science Citation Index.

The Pacific Journal of Mathematics (ISSN 0030-8730) at the University of California, c/o Department of Mathematics, 798 Evans Hall \#3840, Berkeley, CA 94720-3840, is published twelve times a year. Periodical rate postage paid at Berkeley, CA 94704, and additional mailing offices. POSTMASTER: send address changes to Pacific Journal of Mathematics, P.O. Box 4163, Berkeley, CA 94704-0163.

PJM peer review and production are managed by EditFLOW ${ }^{\circledR}$ from Mathematical Sciences Publishers.

\section{PUBLISHED BY}

mathematical sciences publishers

nonprofit scientific publishing

http://msp.org/

(C) 2013 Mathematical Sciences Publishers 


\section{PACIFIC JOURNAL OF MATHEMATICS}

Volume $266 \quad$ No. $1 \quad$ November 2013

Multiplicity of solutions to the Yamabe problem on collapsing $\quad 1$ Riemannian submersions

Renato G. Bettiol and Paolo Piccione

Rank gradient of small covers

DARLAN GIRÃO

Nonrationality of nodal quartic threefolds

KYUSIK HONG

Supertropical linear algebra

ZUR IZHAKIAN, MANFRED KNEBUSCH and LOUIS ROWEN

Isometry groups among topological groups

PIOTR NIEMIEC

Singularities and Liouville theorems for some special conformal

Hessian equations

QIANZHONG OU

Attaching handles to Delaunay nodoids

FRANK PACARD and HAROLD ROSENBERG

Some new canonical forms for polynomials

BRUCE REZNICK

Applications of the deformation formula of holomorphic one-forms 